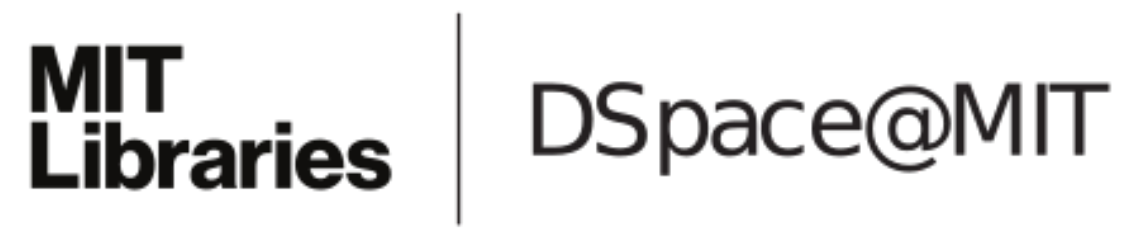

\author{
MIT Open Access Articles \\ Whose Mass is it Anyway? Particle \\ Cosmology and the Objects of Theory
}

The MIT Faculty has made this article openly available. Please share how this access benefits you. Your story matters.

Citation: Kaiser, D. "Whose Mass is it Anyway? Particle Cosmology and the Objects of Theory." Social Studies of Science 36, no. 4 (August 1, 2006): 533-564.

As Published: http://dx.doi.org/10.1177/0306312706059457

Publisher: Sage Publications

Persistent URL: http://hdl.handle.net/1721.1/84945

Version: Author's final manuscript: final author's manuscript post peer review, without publisher's formatting or copy editing

Terms of Use: Article is made available in accordance with the publisher's policy and may be subject to US copyright law. Please refer to the publisher's site for terms of use. 


\title{
Whose Mass is it Anyway? Particle Cosmology and the Objects of Theory
}

\author{
David Kaiser
}

\begin{abstract}
Physicists in different branches of the discipline were puzzled by the problem of mass during the 1950s and 1960s: Why do objects have mass? Around the same time, yet working independently, specialists in gravitational studies and in particle theory proposed that mass might arise due to objects' interactions with a new (and as yet undetected) field. Although the questions they posed and even the answers they provided shared several similarities - and even though both proposals quickly became 'hot topics' in their respective subfields - virtually no one discussed one proposal in the light of the other for nearly twenty years. Only after massive, unprecedented changes in pedagogical infrastructure rocked the discipline in the early 1970s did a new generation of physicists begin to see possible links between the Brans-Dicke field and the Higgs field. For the new researchers, trained in different ways than most of their predecessors, the two objects of theory were not only similar - some began to proclaim that they were exactly the same. Charting the histories of these two objects of theory illuminates the complicated institutional and pedagogical factors that helped to produce a new subfield, particle cosmology, which today ranks at the very forefront of modern physics.
\end{abstract}

Keywords: pedagogy, Higgs field, Brans-Dicke field, particle physics, cosmology 


\section{The Objects of Theory}

Particle cosmology, a hybrid endeavor investigating the smallest units of matter and their role in determining the shape and fate of the entire universe, is among the hottest of hot topics in modern physics today. It has become a staple topic for Scientific American articles and NOVA television specials. (See, for example, Choi, 2004; Dvali, 2004; Krauss \& Turner, 2004; Lloyd \& Ng, 2004; Veneziano, 2004; and WGBH, 1999, 2000, 2003.) During 2004 it received more than half a billion dollars from the U.S.

Department of Energy, the National Science Foundation, and NASA. Almost two new preprints on the topic are posted to the world's central electronic physics preprint server every hour of every single day (for a review, see Guth \& Kaiser, 2005). ${ }^{1}$

A common story is frequently repeated to explain how particle cosmology emerged and grew to its present-day predominance: exciting new developments within particle theory during the mid-1970s, according to this account, convinced many particle theorists to turn their attention to higher and higher energies. They quickly realized that the new super-high energy regime of interest could never be replicated experimentally on earth (at least not using conventional approaches to particle accelerators), but that such energies had existed at one time, during the first fractions of a second after the big bang. Hence particle theorists naturally turned to cosmology, and - presto chango - the new subfield was born.

This story has been repeated ad nauseam in physicists' review articles and popular accounts, and in coverage by science journalists and even some historians and philosophers of science. It certainly captures an important element of the emergence of particle cosmology. But is it sufficient for explaining the birth of this new - and now dominant - branch of the discipline? As significant as the changes within particle theory were, I contend that to fully understand the rapid changes in physics we must look beyond the realm of ideas alone. Rather, we must take into account the concrete 
exigencies of pedagogical infrastructure, especially in the United States. The 'marriage' of particle theory with gravitation and cosmology can only be understood by investigating changes in institutions and training.

One revealing way into this material is to follow the fortunes of theorists' changing objects of study: the objects of theory. Elsewhere I have urged that historians, philosophers, and sociologists should focus on more than just full-blown theories or paradigms in order to understand developments in theoretical sciences; we must look carefully at theorists' tools and at the training required for new recruits to wield those tools in actual research (Kaiser 1998; Kaiser et al., 2004; Kaiser, 2005a, 2005b; see also Olesko, 1991; Pickering \& Stephanides, 1992; Galison \& Warwick, 1998; Klein, 2001, 2003; Kennefick, 2000; MacKenzie, 2003; Warwick, 2003). Here I focus on a related but distinct element of theorists' practice - equally removed from the discourse of theories, paradigms, and conceptual worldviews. Following recent work on the 'biographies of scientific objects', I suggest that we trace the life course of objects of theory: what counted as a proper object of study for theorists at various times and places, and how were these objects pedagogically conditioned? Which objects of theory were deemed salient by various communities, and how did the understandings or associations crafted for these objects change? Note that the problematic is distinct from the philosophers' well-worn analyses of entity-realism and the like; the question is not whether or when scientists, historians, or philosophers may be justified in ascribing reality to a given theoretical entity. Rather, the analysis focuses on the conceptually prior question: why have certain objects commanded scientific attention in the first place? (Arabatzis, 1995, 1996; Daston, 2000; see also Hacking, 1983: part A).

A fruitful entrée into some of the larger-scale changes in physics, and the emergence of particle cosmology in particular, is provided by following a pair of these objects of theory during the second half of the twentieth century: the Brans-Dicke field and the Higgs field. These two objects provide a remarkable barometer for charting 
disciplinary dynamics, revealing how intertwined intellectual and institutional developments can become. Thus to begin to unpack the complicated early history of particle cosmology, we must turn to a problem that exercised many physicists in the late 1950s and early 1960s: the problem of mass.

\section{The Problem of Mass: A Tale of Two $\varphi$ 's}

Physicists in at least two branches of the discipline faced an important question in the 1950 s and early 1960s: Why do objects have mass? Mass seems like such an obvious, central property of matter that one might not even think it requires an explanation. Yet finding descriptions of the origin of mass that remained compatible with other ideas from modern physics proved no easy feat (Jammer, 1961, 2000). The problem of mass took different forms within the two communities of specialists who came to focus on it at mid-century. Experts on gravitation and cosmology framed the problem in terms of Mach's principle. Mach's principle - named for the physicist and philosopher Ernst Mach (1838-1916), famed critic of Newton and inspiration to the young Einstein - remains stubbornly difficult to formulate, but a good approximation might be phrased this way: Are local inertial effects the result of distant gravitational interactions? In other words, does an object's mass - a measure of its resistance to changes in its motion - ultimately derive from that object's gravitational interactions with all the other matter in the universe? If so, do Einstein's gravitational field equations, the governing equations of general relativity, properly reflect this dependence? ${ }^{2}$

Within the much larger community of specialists in particle physics, the problem of mass arose in a different form: how could theorists incorporate masses for elementary particles without violating the required symmetries of their equations? Beginning in the 1950s, particle theorists found that they could represent the effects of nuclear forces by imposing special classes of symmetries (invariance under certain 'gauge 
transformations') on their equations governing sub-atomic particles' behavior. Yet the terms they would ordinarily include in these equations to represent particles' masses violated these special symmetries. Among many types of particles, this impasse affected the force-carrying particles thought to give rise to various nuclear forces. If these particles were truly massless, then the range of nuclear forces should have been infinite two protons should have been able to exert a nuclear force on each other from across a room, or indeed from across the solar system or galaxy. Such a long range for nuclear forces was in flagrant contradiction to the forces' observed behavior, which fell off rapidly for distances larger than the size of atomic nuclei. Only if the force-carrying particles had some mass would the effective range of the nuclear forces come into line with observations. The same basic problem affected the mass-terms for practically all sub-atomic particles when treated in the new gauge field theories: even for gardenvariety particles like electrons, physicists remained unable to write down self-consistent terms for these particles' non-zero masses without violating the gauge symmetries they wanted to impose on their governing equations. The origin of mass thus remained no small problem for particle theorists: they could either represent the forces' symmetries but lose all ability to match basic observations, or they could account for the particles' masses but lose all ability to represent the symmetry properties of sub-atomic forces (see, for example, Brown et al. 1989; Brown \& Rechenberg, 1996; Hoddeson et al. 1997).

At roughly the same time, physicists in both fields proposed explanations of the origin of mass. Both proposals postulated that a new scalar field existed in the universe, whose interactions with all other types of matter explained why we see those other objects as possessing mass. ${ }^{3}$ On the gravitation side, Princeton graduate student Carl Brans and his thesis advisor, Robert Dicke, pointed out in a 1961 article that in Einstein's general relativity, the strength of gravity was fixed once and for all by Newton's constant, $G$. According to Einstein, $G$ had the same value on earth as it did in the most distant galaxies; its value was the same today as it had been billions of years ago. In place of 
this, Brans and Dicke asked what would happen if Newton's gravitational constant varied over time and space. To make this variation concrete, they introduced a new scalar field, $\varphi$, inversely proportional to Newton's constant: $G \sim 1 / \varphi(x)$. Starting from the standard equations of Einstein's general relativity, Brans and Dicke substituted $1 / \varphi$ in place of $G$. Next they noted that if the field $\varphi$ varied over time or space it would carry kinetic energy, and this new source of energy would have to be accounted for in their governing gravitational equations as well. Combining these steps led to their new gravitational equations (Brans, 1961; Brans \& Dicke, 1961). ${ }^{4}$

Beginning from the Brans-Dicke equations rather than the Einsteinian set modified the behavior of ordinary matter: now it responded both to the curvature of space and time, as in ordinary general relativity, and to the variations in the local strength of gravity, coming from $\varphi$. Brans and Dicke introduced a dimensionless constant, $\omega$, in front of $\varphi$ 's kinetic-energy term as a kind of lever to control how strongly the quantitative predictions of their new gravitational equations would differ from the usual Einsteinian case. If $\omega$ were small (on the order of 1 or 10 ), then it would cost relatively little energy for $\varphi$ to vary appreciably over time or space, and hence predictions from their modified gravitational equations would differ measurably from Einstein's equations. But as $\omega$ were made larger, it would become more and more difficult for $\varphi$ to vary; its derivatives would remain small and it would approximate a constant value everywhere. In the limit as $\omega \rightarrow \infty$, then, $\varphi$ (and hence $G$ ) would behave as a constant, returning to the original Einsteinian formulation. So compelling did the new Brans-Dicke work seem that members of Kip Thorne's gravity group at the California Institute of Technology used to joke that they believed in Einstein's general relativity on Mondays, Wednesdays, and Fridays, and in Brans-Dicke gravity on Tuesdays, Thursdays, and Saturdays (they remained agnostic on Sundays, enjoying playtime at the beach) (Will, 1993 [1986]: 156). Physically, the main idea behind the Brans-Dicke work was that some new field, $\varphi$, permeated all of space, and its behavior was determined by the distribution of matter and 
energy throughout the universe. All matter interacted with the new field, and thus $\varphi$ 's behavior helped to determine how ordinary matter would move through space and time. Any measurements of an object's mass would therefore depend on the local value of $\varphi$ (Brans 1961; Brans \& Dicke, 1961; Dicke, 1964a, 1964b).

On the particle theory side, several researchers attacked the problem of mass with a new scalar field at the same time. Yoichiro Nambu, Jeffrey Goldstone, François Englert, Robert Brout, Philip Anderson, T. W. B. Kibble, Peter Higgs and others all focused on the topic during the late 1950s and early 1960s. Building on an analogy to superconducting systems, Jeffrey Goldstone noted in 1961 that equations' solutions need not obey the same symmetries that the governing equations do. As a simple illustration he introduced a scalar field, $\varphi$, whose potential energy density, $V(\varphi)$, behaved as in Figure 1. This potential has two minima, one at a value of $-v$ for the field $\varphi$, and one at the value of $+v$.

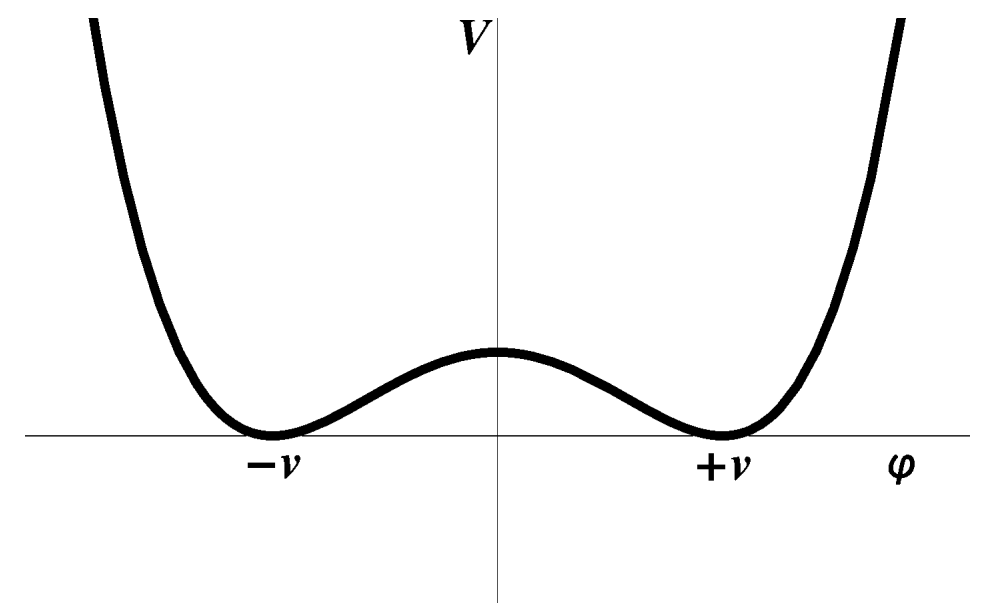

Figure 1. Double-well potential, $V(\varphi)$. The energy of the system has a minimum when the field reaches the values $+v$ or $-v$. Although the field's potential energy is symmetric, the field's solution will pick out only one of these two minima, breaking the symmetry of the governing equations.

The energy of the system is lowest at these minima, and hence the field will eventually settle into one of these values. The potential energy is exactly the same for both of these values of the field; the governing equations are symmetric for either solution, whether $\varphi$ 
winds up at $-v$ or $+v$. Yet the field must eventually land at only one of these values: it has an equal chance of winding up at either $-v$ or $+v$, but it can't end up at both values. The field's solution thus spontaneously breaks the equations' symmetry: whereas $V(\varphi)$ is fully left-right symmetric, any given solution for $\varphi$ would be concentrated only on the left or only on the right (Goldstone, 1961; see also Brown \& Cao, 1991; Brown et al. 1997). ${ }^{5}$

In 1964, Scottish theorist Peter Higgs revisited Goldstone's work, showing that when applied to gauge field theories, spontaneous symmetry breaking would yield massive particles. The key was that the governing equations would necessarily contain terms representing the interaction of the new scalar field, $\varphi$, with the force-carrying gauge fields. These interaction terms obeyed all of the required symmetries, leaving the overall equations unchanged even after a gauge transformation. Yet once the scalar field $\varphi$ settled into one of the minima of its potential, either $+v$ or $-v$, these interaction terms would behave just like a mass: the particles of the gauge field would acquire a non-zero mass given by $m^{2}=2 g^{2} v^{2}$, where $g$ was a coupling constant that determined the strength with which the two fields interacted. The force-carrying particles thus would acquire mass in any particular solution to the governing equations, even though no such masses appeared in the governing equations themselves (Higgs, 1964a, 1964b, 1966). ${ }^{6}$ Similar interactions would link $\varphi$ to the fields for ordinary matter, such as electrons, likewise generating masses for these particles once $\varphi$ settled into a minimum of its potential. Physically, the picture that emerged from the Higgs mechanism (as this procedure quickly came to be called) was that the Higgs field, $\varphi$, permeated all of space, and elementary particles constantly interacted with it. Once $\varphi$ settled into one of its final states, the motions of all other particles would be affected: it would be as if they now moved through some viscous medium rather than empty space. In other words, they would behave as if they had a non-zero mass, and any measurements of the mass would depend on the local value of $\varphi$. 
Both sets of papers - by Brans and Dicke, and by Higgs - quickly became 'renowned', acquiring more than 500 citations by 1981; to this day, each of these papers remains within the top $0.1 \%$ most-cited physics articles of all time. ${ }^{7}$ (See Fig. 2.)
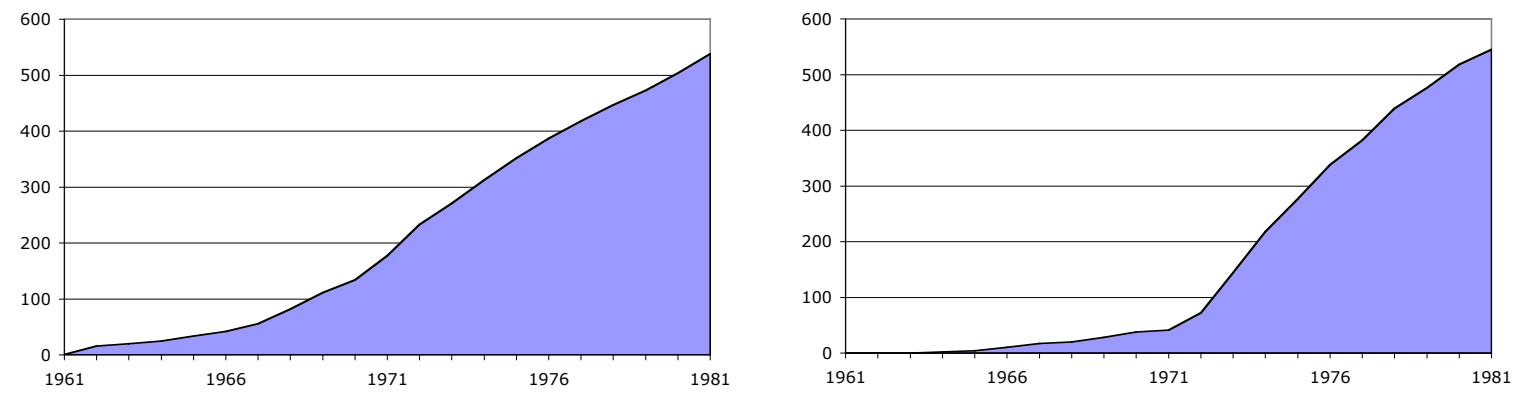

Figure 2. Cumulative citations to the Brans-Dicke (left) and Higgs (right) articles, 1961-81. Based on data in Science Citation Index.

Both papers proposed to explain the origin of mass by introducing a new scalar field, $\varphi$, and accounting for its interactions with all other types of matter. Both were published around the same time, with lengthy articles appearing in the same journal, the Physical Review. Given the similarity of their proposals and the quick attention that both received, one might have expected physicists to consider them alongside one another. ${ }^{8}$

Yet this almost never happened. The two theoretical objects - the Brans-Dicke field, $\varphi_{B D}$, and the Higgs field, $\varphi_{H}$ - serve as tracers, marking out the stark boundaries that existed at the time between the subfields of gravitation and cosmology on the one hand and particle physics on the other. Figure 2 represents 1083 papers that cited either the Brans-Dicke paper or the Higgs papers between 1961 and 1981. Only 6 of theseless than $0.6 \%$ - cited both Brans-Dicke and Higgs, the earliest in 1972 and the rest after 1975. (Although Goldstone's 1961 article received 487 citations between 1961 and 1981, only one paper cited both Brans-Dicke and Goldstone during this period.) Another measure of these communities' separation comes from their author pools: only 21 authors out of the 990 represented in Figure 2 cited both the Brans-Dicke article and Higgs's work - usually in separate papers - between 1961 and 1981. Clearly the two 
communities saw different things in their respective $\varphi$ 's. To the experts in gravitation and cosmology, $\varphi_{B D}$ was exciting because it offered an alternative to Einstein's general relativity, inspiring renewed theoretical scrutiny of gravitational equations and spurring high-precision experimental tests of gravitation. To the particle theorists, $\varphi_{H}$ was exciting because it offered hope that gauge field theories might be able to explain the behavior of nuclear forces among massive particles. Nobody suggested that $\varphi_{B D}$ and $\varphi_{H}$ might be physically similar, or even worth considering side by side, before the mid1970s. ${ }^{9}$

Why so little overlap? Were the objects, $\varphi_{B D}$ and $\varphi_{H}$, fundamentally different from each other, or even 'incommensurable'? Certainly not in any absolute or transhistorical sense: as we will see below, in the mid- and late 1970s a few theorists began to suggest that $\varphi_{B D}$ and $\varphi_{H}$ might be physically identical, literally the same field. Within a few years, several other groups had begun to explore the cosmological consequences of models that included both a Brans-Dicke field and a Higgs field, often merging the two $\varphi$ 's into one. So the objects' changing status cannot be a function only of the things in themselves. The objects' status and identity were historical. A maneuver that no one had even considered in the 1960s and early 1970s became an obvious, run-of-the-mill procedure by the early 1980s. What had changed between the mid-1960s and the late 1970s that might account for physicists' remarkably different treatment of these two objects of theory?

\section{Pushes, Pulls, and Pedagogy}

The separate treatment of the two objects of theory, $\varphi_{B D}$ and $\varphi_{H}$, illuminates the wide gulf that separated specialists in gravitation from those in particle physics throughout the 1960s. The objects also reveal some of the more subtle contours within the world's communities of theoretical physicists: not all groups treated the two $\varphi$ 's the 
same way, or along similar time-lines. Yasunori Fujii, for example, a young Japanese theorist working in Tokyo, first tentatively suggested that $\varphi_{B D}$ and $\varphi_{H}$ might be one and the same field as early as 1974 , and over the next four years similar proposals were broached by theorists working in Kiev, Brussels, and Bern (Fujii, 1974; Englert et al. 1975; Minkowski, 1977; Matsuki, 1978; Chudnovskii, 1978). Yet no one working or trained within the United States considered $\varphi_{B D}$ and $\varphi_{H}$ on an equal footing (and exceedingly few considered the two fields together at all) until 1979. The divide between particle physics and cosmology was especially sharp in the United States, and only began to subside after massive changes in pedagogical infrastructure rocked the field in the early 1970s. These dramatic institutional changes helped set the conditions for a new generation of American-trained physicists to begin to treat the two $\varphi$ 's as mutually relevant.

During the middle decades of the twentieth century, general relativity and cosmology lost much of the allure they had once held among physicists around the world (Eisenstadt, 1989; Kaiser, 1998). Yet the division between gravitation and cosmology and 'mainstream' topics within physics never became as stark in many countries as it did within the United States. When composing their renowned textbook series on theoretical physics during the 1940s and 1950s, for example, the Moscow theorists Lev Landau and Evgenii Lifshitz incorporated general relativity and cosmology into the heart of their book on classical field theory - and hence Einstein's theory of gravity was interwoven with such staple topics as electromagnetism. Undergraduates and graduate-level physics students at Cambridge University likewise continued to study general relativity as part of their ordinary coursework during the 1940s and 1950s, much the way their predecessors had been doing since the 1920s. Meanwhile several Swiss-based physicists launched a new 'International Society on General Relativity and Gravitation' in 1961. ${ }^{10}$

Nor had the division between high-energy physics and gravitation always been so severe among physicists working in the United States. During the 1930s, several 
physicists in the United States contributed to the new field of 'relativistic astrophysics'. For example, J. Robert Oppenheimer and some of his students at Berkeley began to investigate the nuclear reactions within massive stars and the space-time structures surrounding them, while Richard Tolman at the California Institute of Technology produced pioneering work on how to study high-temperature phenomena in the context of general relativity (Oppenheimer \& Serber, 1938; Oppenheimer \& Volkoff, 1939; Oppenheimer \& Snyder, 1939; Tolman, 1934). Subrahmanyan Chandrasekhar arrived at the University of Chicago's Yerkes Observatory from Cambridge University in 1937, and began to build up a thriving school of young astrophysicists who worked with him to investigate relativistic stellar structure and dynamics (Chandrasekhar, 1939, 1942; see also Kragh 1999: 183-84; Srinivasan, 1999). A decade later George Gamow and his young collaborators began combining insights from nuclear physics with relativistic cosmology to piece together what came to be known as the 'big bang' model of the universe (Alpher, 1948; Alpher, Bethe \& Gamow, 1948; Alpher, Herman \& Gamow, 1948; Alpher \& Herman, 1948, 1949, 1950; Gamow, 1948a, 1948b, 1949; see also Kragh, 1996: chap. 3). Yet unlike the earlier work, Gamow and company's efforts received nary a passing glance from their American colleagues: discounting selfcitations, the cluster of articles published between 1948 and 1950 by Gamow's group received an average of fewer than three citations per year in the American physics literature over the next fifteen years. Many of these citations, meanwhile, came from physicists working outside the United States who chose to publish in the American journals. $^{11}$

When most American physicists turned with renewed vigor (and off-scale funding) to nuclear and particle physics after World War II, questions of gravitation and cosmology quickly fell by the wayside. New machines beckoned, capable of accelerating sub-atomic particles to higher and higher energies, and the great majority of particle theorists working in the United States set as their goal trying to bring some order to the 
'zoo' of unanticipated new particles and interactions that began to stream forth (Pickering, 1984; Polkinghorne, 1989; Brown et al., 1989; Galison, 1987, 1997). Even the most abstract-minded of the postwar generation of American particle theorists preferred to keep their focus on the new data and on increasingly fancy ways of representing the new particles and their interactions. General relativity and cosmology fell out of their students' curricula; many American physicists took to mocking such 'speculative' topics, so far had they fallen outside mainstream developments (Pickering, 1984; Kaiser, 2005a; Schweber, 1989). ${ }^{12}$

Well into the 1960s, the divide in the United States between particle physics and gravitation and cosmology remained stark. When planning for the future, American physicists saw distinct trajectories for the two fields. The Physics Survey Committee (PSC) of the National Academy of Sciences published a lengthy study in 1966, Physics: Survey and Outlook, meant to provide 'a report on the present state of U.S. physics and its requirements for future growth'. Although particle physics received the single largest share of federal funding among the subfields of physics in the mid-1960s, the PSC recommended more than doubling that amount by 1969 - the largest increase suggested for any subfield within physics. Similarly, they called for the number of postdoctoral researchers working full-time in particle physics to double between 1966 and 1972, from one thousand to two thousand. Meanwhile they made no specific recommendations for amplifying gravitational or cosmological research, and called for rather modest increases in astrophysics generally: the report recommended that astrophysics should receive the second smallest increase in federal funds among all the subfields of physics, and further suggested that the number of Ph.D.-level practitioners rise from 590 to 670 - a $14 \%$ increase, the lowest among all subfields by more than a factor of three (PSC, 1966: 38$45,52,95,111)$. Within the United States, the view from the mid-1960s seemed clear: particle physics should continue its steep upward climb while gravitation and cosmology remained separate, small, and dormant. 
As the treatment of $\varphi_{B D}$ and $\varphi_{H}$ makes clear, however, by the late $1970 \mathrm{~s}$ the separation between cosmology and particle physics no longer seemed quite so extreme, even among physicists working in the United States. Looking back on the rapid rise of particle cosmology, physicists almost always point to two important developments that helped spur the merger. Both concerned changes in particle theory during the mid-1970s: the discovery of 'asymptotic freedom' in 1973, and the construction of the first 'grand unified theories', or GUTs, in 1973-74. Asymptotic freedom refers to an unexpected phenomenon within certain classes of gauge field theories: the strength of the interaction decreases as the energy of the particles goes up, rather than increasing the way most other forces do. For the first time, particle theorists were able to make accurate and reliable calculations of such phenomena as the 'strong nuclear force' - the force that keeps quarks bound within nuclear particles such as protons and neutrons - as long as they restricted their calculations to very high energy realms, far beyond anything that had been probed experimentally (Gross \& Wilczek, 1973a, 1973b, 1974; Politzer, 1973, 1974). (H. David Politzer, David Gross, and Frank Wilczek shared the 2004 Nobel Prize in Physics for their discovery of asymptotic freedom.) The introduction of GUTs likewise pointed particle theorists' attention toward very high energies. Some particle theorists realized that the strengths of three of the basic physical forces - electromagnetism, the weak nuclear force (responsible for such phenomena as radioactive decay), and the strong nuclear force - might become equal at some very high energy. (See Fig. 3.) 


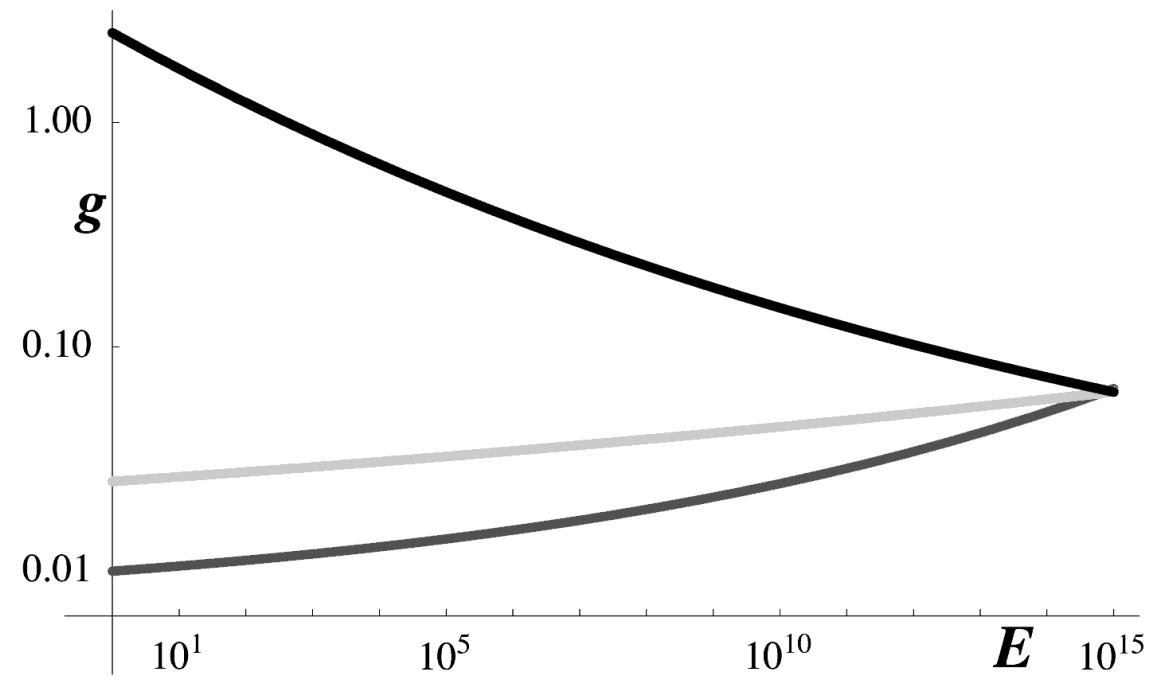

Figure 3. Interaction strength ( $g$, in arbitrary units) versus energy ( $E$, in billions of electron-volts). From top to bottom: the interaction strengths associated with the gauge groups representing the strong nuclear force, the weak nuclear force, and electromagnetism. Note that the interaction strength of the strong nuclear force decreased with increasing energy ('asymptotic freedom'), while those of the weak and electromagnetic forces increased with energy, allowing all three interaction strengths to intersect in the vicinity of $10^{15}$ billion electron-volts.

Theorists hypothesized that above that energy scale the three forces would act as a single undifferentiated force, subject to a particular gauge symmetry group. Below that energy scale, the GUT symmetry would be spontaneously broken, leaving three distinct gauge groups, each with its characteristic interaction strength (Georgi \& Glashow, 1974; see also Pati \& Salam, 1973).

The energy scale at which 'grand unification' might set in was literally astronomical: more than one trillion times higher than anything particle physicists had been able to probe using earth-bound particle accelerators. Physicists had no possible way of accessing such energy scales via their traditional route; even with three decades of improvements in the underlying technology, today's most powerful particle accelerators have increased the energies under study by less than a factor of one hundred, a far cry from one trillion. So GUT-scale energies could never be created in physicists' laboratories. But some began to realize that if the entire universe had begun in a hot big bang, then the average energy of particles in the universe would have been 
extraordinarily high at early times in cosmic history, cooling over time as the universe expanded. With the advent of asymptotic freedom and GUTs, particle physicists therefore had a 'natural' reason to begin asking about the high-energy early universe: cosmology would provide 'the poor man's accelerator'. Scores of physicists, journalists, philosophers, and historians have repeated this refrain to explain the birth of particle cosmology: key ideas within particle theory drove particle theorists to think about cosmology, beginning in the mid-1970s. ${ }^{13}$

Is this the whole story? Although certainly important, these changes in particle theory are not sufficient to explain the growth of the new subfield. For one thing, the timing is a bit off. Publications on cosmology (worldwide as well as in the United States) began a steep rise before 1973-74, and the rate of increase was completely unaffected by the appearance of the papers on asymptotic freedom and GUTs. (See Fig. 4.)

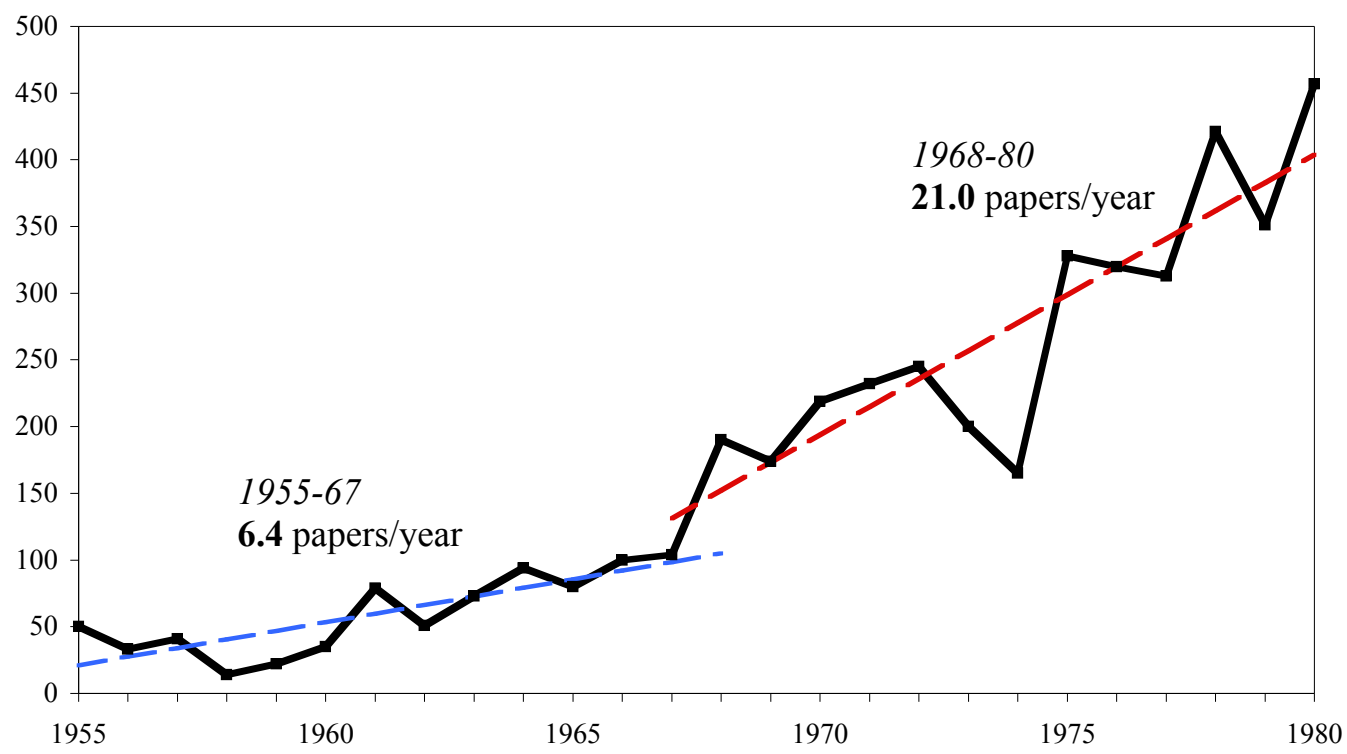

Figure 4. Number of papers published worldwide on cosmology per year. Dashed lines show average rates of growth during the two periods. Based on data in Physics Abstracts. 
Whereas the annual number of papers worldwide on cosmology grew at an average pace of 6.4 papers per year between 1955 and 1967, this rate soared to 21 additional papers per year between 1968 and 1980. Within the main American research journal, the Physical Review, the rate at which cosmology articles appeared similarly quadrupled between the periods 1960-67 and 1968-73. ${ }^{14}$ Moreover, although GUTs were introduced in 1973-74, they did not receive much attention - even from particle theorists - until the late 1970s and early 1980s. ${ }^{15}$ Three of the earliest review articles on the emerging field of particle cosmology, published between 1978 and 1980, highlighted strictly non-GUT-related work, some dating back to 1972 before either asymptotic freedom or GUTs had even been introduced (Schramm, 1978; Steigman, 1979; Tayler, 1980). ${ }^{16}$ By the late 1970s, several reviewers thus recognized the new subfield of particle cosmology independent of asymptotic freedom or grand unification; the new ideas from particle theory surely did not create the subfield all on their own.

More than ideas were at stake in the creation of particle cosmology: institutions and infrastructure played major roles as well. Détente, major cutbacks in defense spending, anti-Vietnam War protests, and the Mansfield Amendment (which heavily restricted Defense Department spending on basic scientific research) wreaked havoc on physics in the United States between 1968 and 1972. The first 'Cold War bubble' - akin to a speculative stock-market bubble, which had seen physicists funded and admired like no other period in American history - burst suddenly, and physicists in the United States quickly began to talk of the crisis facing their discipline (Kaiser, 2002: 149-153). ${ }^{17}$ The overall number of physics Ph.D.s granted per year in the United States entered a steep decline, falling nearly as fast during $1970-75$ as it had risen during the years after Sputnik. (See Fig. 5. ${ }^{18}$ ) 


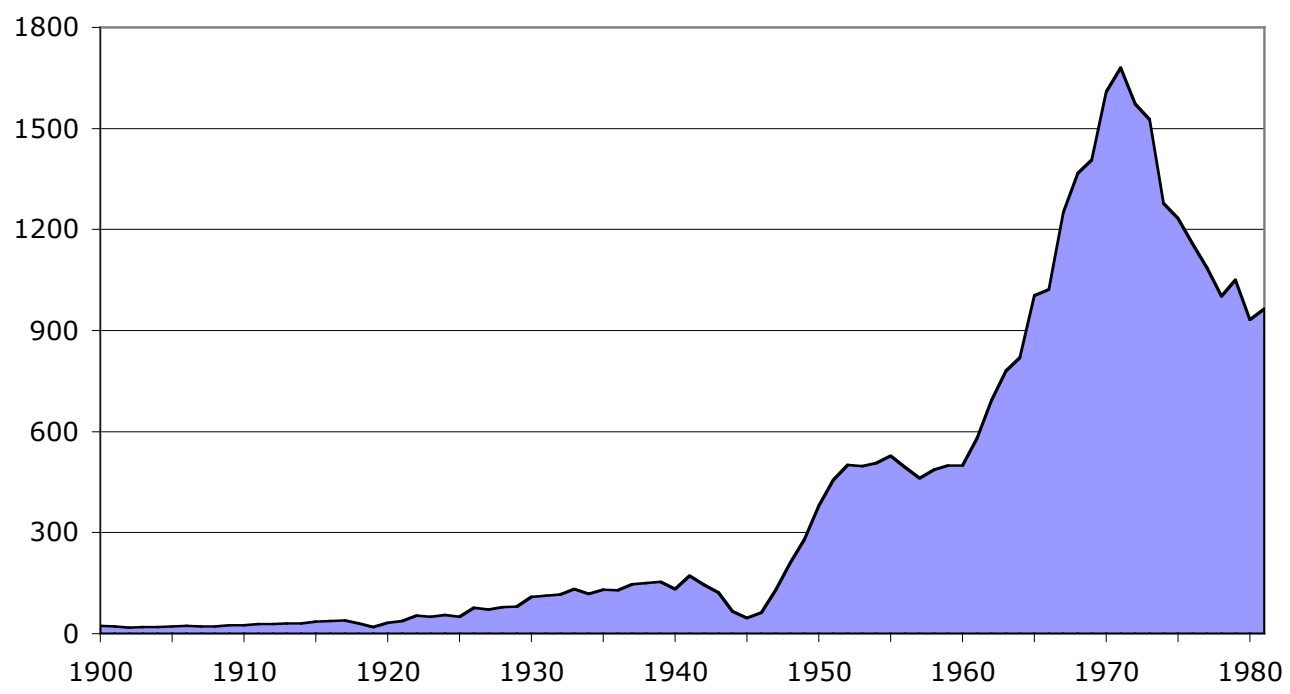

Figure 5. Number of physics Ph.D.s granted per year in the United States, 1900-81.

Federal funds for physics likewise fell rapidly, down by more than one-third between 1967 and 1976 (in constant dollars). Whereas employers had always outnumbered physics students looking for jobs at the American Institute of Physics's placement service meetings from the 1950s through the mid-1960s, employment prospects quickly turned grim for young physicists in the United States: 989 applicants competed for 253 jobs in 1968, while 1,053 sought out only 53 jobs on offer in $1971 .^{19}$ By the early 1970 s, physicists in the United States faced the worst crisis their discipline had ever seen.

The cuts did not fall evenly across the discipline: hardest hit by far was particle physics. Federal spending on particle physics fell by 50\% between 1970 and 1974 (a combination of direct cut-backs and inflation), combined with a sudden drop in government demand for high-energy physicists (Kevles, 1995 [1978]: 421). A rapid outflow of particle physicists began: between 1968 and 1970 alone, twice as many physicists left particle physics as entered it in the United States. The downward slide continued into the 1970s: the number of new particle physics Ph.D.s trained per year in the United States fell by $44 \%$ between 1969 and 1975 - the fastest decline of any subfield 
- and the proportion of new Ph.D.s entering particle physics fell by more than a third (PSC, 1972, vol. 1: 367; PSC, 1986: 98). As particle physicists' fortunes plummeted, meanwhile, astrophysics and gravitation became one of the fastest-growing fields in American physics. Spurred in part by a series of new discoveries during the mid-1960s (such as quasars, pulsars, and the cosmic microwave background radiation), as well as by innovations in experimental and instrumental approaches to gravitation and astrophysics, the number of new Ph.D.s per year in this area grew by $60 \%$ between 1968 and 1970, and by another 33\% between 1971 and 1976 - even as the total number of physics Ph.D.s fell sharply. ${ }^{20}$ All told, the number of dissertations on gravitation and cosmology within the United States grew twice as fast between 1968-80 as they had between 1955-67. ${ }^{21}$

Surveying the wreckage a few years into the slump, the Physics Survey Committee (PSC) argued that particle theorists (who made up more than half of all the new Ph.D.s in particle physics) had fared so poorly when the crunch came because their training had been too narrowly specialized. When demand for particle physicists fell off, too many of the young particle theorists had difficulty switching their research efforts elsewhere. The nation's physics departments needed to revamp how particle theorists were trained, urged the PSC:

\footnotetext{
The employment problem for theoretical particle physicists appears to be even more serious than it is for other physicists. The large number of such theorists produced in recent years and their high degree of specialization are often given as the causes of this difficulty. This narrow specialization is already an indication that the student of particle theory has been allowed to choose unwisely, because real success in any part of physics requires more breadth, and both great breadth and depth of perspective are required for a significant contribution, especially in theoretical particle physics. ... University groups have a responsibility to expose their most brilliant and able students to the opportunities in all subfields of physics. (PSC, 1972, vol. 1: 119)
}

Particle theorists were the only subfield singled out for such criticism in the PSC's 2500page report. Particle physicists in the United States did undertake curricular changes in the early 1970s, aimed in part to broaden their students' exposure to other areas of 
physics - including more emphasis on gravitation and cosmology. Across the country, physics departments began to offer new courses on the subject.

One useful measure of these curricular changes comes from the fast-rising numbers of graduate-level textbooks on gravitation and cosmology. Soon after the end of World War II, with graduate physics enrollments skyrocketing like never before, American publishers realized that advanced physics textbooks could be a lucrative business. A dozen presses aggressively entered the field, especially eager to publish anything on quantum theory and nuclear physics (Kaiser 2005a: $255-259$ ). ${ }^{22}$ Yet despite the booming enrollments and brisk sales, American publishers shied away from textbooks on gravitation and cosmology after the war - understandably so, since no physics departments in the country required coursework in the subject and most failed even to offer elective classes in it. ${ }^{23}$ The handful of textbooks on gravitation and cosmology available to American physicists during the 1950s and 1960s were dominated by translations of successful, older foreign-language books - books like Hermann Weyl's Space-Time-Matter (1919, first English translation 1950), Landau and Lifshitz's Classical Theory of Fields (1941, first English translation 1951), Wolfgang Pauli's Theory of Relativity (1921, first English translation 1958), and Vladimir Fock's Theory of Space-Time and Gravitation (1955, first English translation 1959). Even as they galloped ahead with scores of new titles in high-energy physics, American publishers and series editors proceeded cautiously when it came to textbooks on gravitation and cosmology. One reviewer of a new textbook manuscript observed in 1959, for example, that 'There is probably not a vast market for a relativity book, however good', and editors and publishers often acknowledged outright that such books probably would never recoup the costs of their production and would have to be subsidized by the other, more successful textbooks in a given series. ${ }^{24}$

With increasing excitement about some of the new gravitational discoveries during the mid- and late 1960s, publishers became a bit less queasy about the subject and 
the number of new textbooks grew modestly. The real take-off in gravitational publishing, however, came in the early and mid-1970s. With the sudden change in fortunes of particle physics, and the growing acknowledgment among many American physics departments that their students needed to gain broader exposure to various topics across theoretical physics, textbooks on gravitation and cosmology finally became a profitable enterprise: American publishers brought out twenty-six new graduate-level textbooks on the subject during the 1970s, and published another six English-language editions of foreign textbooks, averaging more than three new textbooks each year (twice the rate for the previous decade). ${ }^{25}$ Amid the fast-changing curricula, physicists sometimes decided not to wait for formal textbooks to be published. In 1971, for example, Caltech began to circulate mimeographed copies of the lecture notes from Richard Feynman's 1962-63 course on gravitation, while the Reidel publishing company in Boston rushed out Achilleus Papapetrou's informal lecture notes on general relativity in $1974 .^{26}$

Meanwhile several major new textbooks appeared: James Peebles's Physical Cosmology (1971), Steven Weinberg's Gravitation and Cosmology (1972), Charles Misner, Kip Thorne, and John Wheeler's Gravitation (1973), and Stephen Hawking and George Ellis's The Large-Scale Structure of Space-Time (1973). ${ }^{27}$ Other publishers hurried new editions of older textbooks back into print: Dover re-published Peter Bergmann's famous Introduction to the Theory of Relativity in 1976 (long after the original 1942 Prentice-Hall edition had gone out of print); after almost deciding that it couldn't afford to publish Ronald Adler, Maurice Bazin, and Menahim Schiffer's Introduction to General Relativity (1965) because there was no market for it, McGrawHill rushed out a second edition in 1975. The time delay between new editions of more recent textbooks, meanwhile, continued to shrink: Harper and Row first published William Kaufmann's Relativity and Cosmology in 1973 and produced a second edition as early as 1976, while Pergamon published revised and expanded English editions of 
Landau and Lifshitz's popular Classical Theory of Fields in 1971 and 1975, each subsequent edition including more material (and even more important, more homework problems) on relativistic cosmology. With more and more graduate students taking more and more formal courses on gravitation and cosmology, new types of textbooks also began to appear. Four of Kip Thorne's Caltech disciples published the Problem Book in Relativity and Gravitation in 1975, a compendium of 500 advanced problems with solutions (Lightman et al. 1975). Although no problems asked students to unite $\varphi_{B D}$ and $\varphi_{H}$ into a single field, dozens of problems prompted students to practice manipulating scalar fields in the context of general relativity and Brans-Dicke gravitation.

These massive changes in American physics left their mark on the way theorists handled such theoretical objects as $\varphi_{B D}$ and $\varphi_{H}$. Working independently, two American theorists suggested in 1979 that $\varphi_{B D}$ and $\varphi_{H}$ might be one and the same field - this after nearly two decades in which almost no American physicists had even mentioned the two theoretical objects in the same paper, let alone considered them to be physically similar. Anthony Zee and Lee Smolin separately introduced a 'broken-symmetric theory of gravity' by combining the Brans-Dicke gravitational equations with a Goldstone-Higgs symmetry-breaking potential, in effect gluing the two key pieces of $\varphi$ together. In this model the local strength of gravity, governed by Newton's 'constant', $G \sim 1 / \varphi^{2}$, not only could vary over space and time (as in the Brans-Dicke work); its present-day value emerged only after the field, $\varphi$, settled into a minimum of its symmetry-breaking potential, just as in the Higgs work. In this way, Zee and Smolin each could try to explain why the gravitational force is so weak, compared to other forces: when the field settles into its final state, $\varphi= \pm v$, it anchors $\varphi$ to some large, non-zero value, thus pushing $G \sim 1 / v^{2}$ to a small value (Zee, 1979; Smolin, 1979). ${ }^{28}$

Anthony Zee's path to uniting the two $\varphi$ 's illustrates one way in which theorists in the United States wandered into cosmology from particle theory after the collapse of the Cold War bubble. He had worked with gravitation-expert John Wheeler as an 
undergraduate at Princeton in the mid-1960s before pursuing his Ph.D. in particle theory at Harvard, earning his degree in 1970 just as the biggest declines in that area began. As he later recalled, 'At that time I was working on problems far removed from those of cosmology. ... I never heard cosmology mentioned during my graduate school years at all.' After postdoctoral work, Zee began teaching at Princeton. He rented an apartment from a French physicist while on sabbatical in Paris in 1974, and in his borrowed quarters he stumbled upon a stack of papers by European theorists who tried to use ideas from particle theory to explain various cosmological features (such as why our observable universe contains more matter than antimatter). Although he found the particular ideas in the papers unconvincing, the chance encounter reignited Zee's earlier interest in gravitation. Returning from his sabbatical, and back in touch with Wheeler, Zee began to redirect his research interests more and more toward particle cosmology. ${ }^{29}$

Lee Smolin, on the other hand, entered graduate school at Harvard in 1975, just as the curricular changes began to take effect. Unlike Zee, Smolin formally studied gravitation and cosmology as a graduate student alongside his coursework in particle theory - he didn't need to stumble into one area from the other. Smolin worked closely with Stanley Deser (based at nearby Brandeis University), who was visiting Harvard's department during Smolin's first year of graduate study. Deser was uniquely well-placed to help Smolin study the new material: he was originally trained as a particle theorist under Julian Schwinger at Harvard in the early 1950s, and had been among the very first physicists to learn about Peter Higgs's work on symmetry-breaking in gauge field theories during the early 1960s (even encouraging the reluctant Higgs to present a seminar on the new work at Harvard). Deser was also the son-in-law of Swedish theorist Oskar Klein, an early champion of general relativity during the interwar period and among the first to try to mix ideas from gravitation with (then new) quantum field theory. Deser, in turn, was one of very few American theorists who had taken an interest in quantum gravity by the 1960 s - attempting to formulate a description of gravitation that 
would be compatible with quantum mechanics. He was also the very first physicist in the entire world to publish an article that cited both the Brans-Dicke work and the Higgs work (although he treated the two fields quite differently and in separate parts of this 1972 paper). ${ }^{30}$

Smolin's other main advisor was Sidney Coleman, a particle theorist who just a few years earlier had begun teaching the first course on general relativity to be offered in Harvard's physics department for nearly twenty years. During his first year of graduate study, meanwhile, Smolin also took an intense course with visiting professor Gerard 't Hooft on advanced techniques in gauge field theories (many of which 't Hooft had just worked out in the early 1970s, and for which he recently shared the Nobel Prize in 1999). Smolin completed an independent study with Howard Georgi, who had published some of the first articles on GUTs just months before; Smolin also did coursework with Steven Weinberg, scrutinizing how the approach to gravitation and cosmology advanced in the recent Hawking and Ellis textbook (published in 1973) compared and contrasted with the methods in Weinberg's own influential textbook (published in 1972). Building on this curricular preparation Smolin worked on topics in quantum gravity, and suggested that $\varphi_{B D}$ and $\varphi_{H}$ might be one and the same field just as he was finishing his dissertation in $1979 . .^{31}$

Smolin's experiences marked the new routine for his generation of theorists, trained during the mid- and late-1970s to work at the interface of gravitation and particle theory. Theorists like Paul Steinhardt, Michael Turner, Edward 'Rocky' Kolb and others - each of whom, like Smolin, received his Ph.D. between 1978 and 1979 - devoted formal study to gravitation as well as to particle theory in graduate school. Steinhardt began his graduate studies at Harvard in 1974, learning along with Smolin about some ways particle physics and cosmology might be combined. Turner, meanwhile, arrived as a graduate student at Stanford in 1971 expecting to work on particle theory, but found the topic in a 'lull', with 'nothing much going on', as he recalled recently. Disappointed, he 
left graduate school for two years. As the bottom was falling out of traditional particle theory and enrollments were dropping, a few of Stanford's theorists began meeting with a small and informal group of graduate students and postdocs to talk about gravitation and cosmology; no longer would they be tied to particle physics as usual. Soon the department began offering a revamped course on gravitation and cosmology; Turner returned to graduate school after he audited the new course, which he found inspiring. ${ }^{32}$

Rocky Kolb likewise began his graduate studies at a propitious moment. Beginning in 1973 he studied particle theory at the University of Texas at Austin, where he worked primarily with the particle theorist Duane Dicus on studies of the weak nuclear force. In addition to Austin's Center for Particle Theory, where Dicus was based, the department had recently built up its Center for Relativity, newly under the direction of Bryce DeWitt. DeWitt, like John Wheeler, had been one of the very few American physicists who devoted his attention to general relativity as early as the 1950s; he left the University of North Carolina to head up Austin's Center for Relativity in the early 1970s, and Austin quickly began pumping out new Ph.D.s who specialized in gravitation and cosmology. (In fact, in 1975 Austin's department produced one quarter of all the physicists who earned Ph.D.s specializing in gravitation within the United States.) With studies of gravitation locally on the rise, students like Kolb learned from the start to combine their investigations of particle theory with questions inspired by gravitation and cosmology. Interspersed with his early articles on 'mainstream' particle theory, Kolb began to work on astrophysical and cosmological bounds that might limit the number and properties of new particles. $^{33}$

Soon Smolin, Turner, Kolb, Steinhardt, and others were training their own graduate students to work in the new hybrid area. For these young theorists and their growing numbers of students, it became 'natural' to associate $\varphi_{B D}$ and $\varphi_{H}$. Turner, Kolb, and Steinhardt each led groups that pursued further links between $\varphi_{B D}$ and $\varphi_{H}$ during the 1980s, constructing cosmological models in which $\varphi_{B D}$ and $\varphi_{H}$ either appeared side-by- 
side or were identified as one single field. Kolb and Turner went further, helping to establish a new institutional home for the burgeoning subfield: in 1983 they became codirectors of the first center for particle astrophysics, based at the Fermi National Accelerator Laboratory near Chicago. They went on to write the first textbook for the new field of particle cosmology, The Early Universe, which first appeared in 1990 (Kolb \& Turner, 1990; see also Nadis, 2004: 48; Overbye, 1991: 206-211). Steinhardt similarly trained large numbers of graduate students in the new field once he began teaching at the University of Pennsylvania in 1981. With these new institutions and newly-trained teachers in place, a new generation of graduate students began to learn how they might relate $\varphi_{B D}$ and $\varphi_{H}$ (Accetta et al. 1985; La \& Steinhardt, 1989; Holman et al. 1990). ${ }^{34}$ What seemed routine to these young theorists beginning in the late 1970s had never been broached before by physicists working in the United States (and only rarely elsewhere). The objects of theory were pedagogically conditioned.

\section{Objects versus Theories}

Empirical evidence likewise played at best a minor role in encouraging physicists to study $\varphi_{H}$ and $\varphi_{B D}$ together. To date, no direct evidence of the Higgs field, $\varphi_{H}$, has been reported. During the superheated debates over the Superconducting Supercollider - a huge particle accelerator whose construction was begun outside of Dallas, Texas, only to have its funding eliminated by the United States Congress in 1993 - many particle physicists elevated the Higgs field to central importance: the possibility of finding signs of $\varphi_{H}$ became a principal argument in favor of building the Supercollider. One particle physicist, Nobel laureate Leon Lederman, went so far as to dub $\varphi_{H}$ the 'God particle' in the midst of the Supercollider discussions; more recently, the Higgs field and its relations to the cancelled Supercollider have even become the subject of a novel by Herman Wouk (of Caine Mutiny and Winds of War fame). ${ }^{35}$ Although the Supercollider project was 
cancelled and no direct evidence for $\varphi_{H}$ has yet been found, few particle theorists doubt that $\varphi_{H}$ or something very much like it exists and is responsible for the dynamical origin of mass. The object, $\varphi_{H}$, has remained central to particle theorists' investigations.

Like $\varphi_{H}$, no direct evidence has yet been established for the existence of the Brans-Dicke field, $\varphi_{B D}$. This has not been for lack of trying: physicists and astronomers subjected Einstein's general relativity and the Brans-Dicke modification to a series of high-precision tests during the 1960s and 1970s. Robert Dicke himself pioneered one of the new types of experimental tests. Together with his Princeton assistant Mark Goldenberg, Dicke began intense studies of the shape of the sun during the mid-1960s. He realized that if the sun's shape departed significantly from that of a sphere, then the usual predictions of general relativity would need to be modified. In particular, the amount by which the sun's mass would deform the spacetime around it, and hence the amount by which the planet Mercury's closest approach to the sun would shift from orbit to orbit, would differ measurably from Einstein's predictions if the sun were oblate rather than perfectly spherical. If the sun really were oblate, meanwhile, then the Brans-Dicke description of gravity would actually match the observations of Mercury's orbit if the Brans-Dicke parameter, $\omega$, were set to around 5. This was no small matter: the perihelion shift of Mercury had long been seen as one of the most crucial successes of Einstein's general relativity; if the Brans-Dicke model actually matched these observations better than Einstein's own gravitational account did, this would be taken as strong evidence that $\varphi_{B D}$ really did exist and that all matter really interacted with it in the way Brans and Dicke had postulated. Everything hinged on how much the sun's shape deviated from that of a sphere; measuring this difference (if any existed) was notoriously tricky. When one of Dicke's former colleagues, Henry Hill, re-did the solar oblateness measurements in the mid-1970s, his group found a much smaller deviation in the sun's shape (effectively a negligible departure from spherical). This seemed to put Einstein's 
general relativity back on top (Dicke \& Goldenberg, 1967; Hill et al., 1974; see also Will, 1993 [1981]: 181-183; Will, 1993 [1986]: chap. 5; Richman, 1996).

Meanwhile gravitational experts pursued a new generation of high-precision tests during the 1970s. These all centered around timing how long it took electromagnetic signals to travel from the earth to some astronomical object and back. According to general relativity, the time required for signals to travel such distances would be affected by how strongly nearby objects (such as the sun) warped or deformed the spacetime through which the signals traveled. The Brans-Dicke model predicted a similar result, but by an amount that differed from the Einsteinian case by a calculable factor of $\omega$. Experts in the new field of experimental gravitation performed a series of these radar ranging tests, sending signals back and forth to various objects throughout the solar system, including the Mariner spacecraft and the Viking spacecraft lander on Mars. By the mid-1970s, groups began to announce that the ordinary Einsteinian predictions seemed to fit their data more easily than did the Brans-Dicke ones. Put another way, they found that only in the limit of large $\omega$ would the Brans-Dicke predictions be compatible with the new empirical data (since as $\omega$ became larger, the quantitative predictions of the Brans-Dicke equations would shift closer and closer to the Einsteinian ones). The early Mariner tests placed a lower limit on $\omega>10$, while in 1979 the group studying radio echoes from the Viking lander found $\omega>500$ (Reasenberg et al. 1979; see also Will, 1993 [1981]: 173-176; Will, 1993 [1986]: chap. 6; Richman, 1996: 17-19).

Some physicists and historians have argued that these new tests constituted the 'experimental refutation' of Brans-Dicke gravity, which had been 'roundly rejected' by the late 1970s; these new tests, it is said, 'sow[ed] the seeds' for Brans-Dicke gravity's 'demise', delivering the 'lethal blow' to the much-beleagured $\varphi_{B D}$ (Richman, 1996: 1, 17, 19-20; Will, 1993 [1986]: 157). In other words, these commentators have cast the gravitational tests in the idiom of theory selection. Yet to do so obscures a great deal about theorists' everyday practices. While relatively few physicists today might believe 
that the Brans-Dicke theory best describes gravity in the present universe, the object $\varphi_{B D}$ was hardly killed off by the 1970 s experiments. The new generation of theorists - people like Smolin, Steinhardt, Kolb, Turner, and their students, trained from the start to work in particle cosmology - have found dozens of new reasons to study $\varphi_{B D}$, far beyond the original motivations of Brans and Dicke. The Brans-Dicke field has become even more central to many theorists' daily work since its purported 'demise': $\varphi_{B D}$ is now thought to be a generic feature of corrections to low-energy gravitation from quantum gravity; it is central to the phenomenology of superstring theories; and it continues to be exploited routinely in investigations of early-universe alterations to gravity, such as during an inflationary epoch. Citations to the original 1961 Brans-Dicke article actually increased during the 1980s and 1990s (see Fig. 6) - a result that is difficult to understand in terms of 'theory selection'. 36

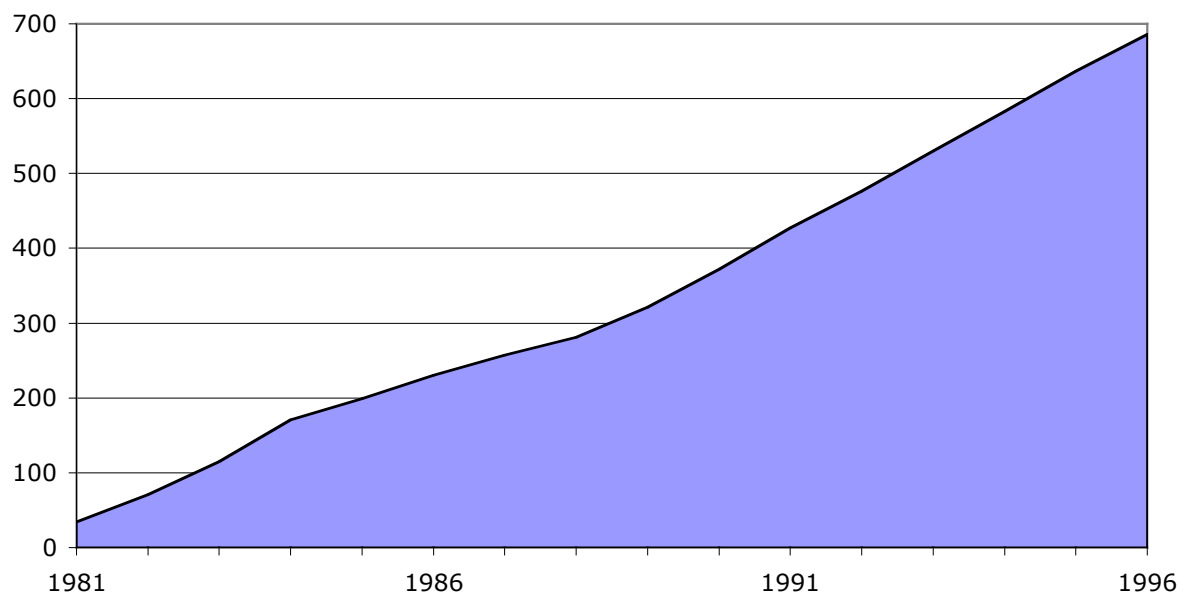

Figure 6. Cumulative citations to the 1961 Brans-Dicke article, 1981-1996. Based on data in Science Citation Index.

The object, $\varphi_{B D}$, in other words, has retained a salience for day-to-day work that is completely missed if one talks only about theories, paradigms, or worldviews. 


\section{Object Lessons}

Theoretical physicists' activities are deeply layered: tools, objects, and theories are not the same thing, nor do they change on the same time-scales. Historians' and philosophers' usual emphasis upon 'theory selection' thus misses a great deal of the everyday labor of theory: tools and objects often live on, plastic and malleable, long after a given theory has supposedly been rejected. As with theorists' uses of tools, the objects of theory rise and fall with specialization and training (Olesko, 1991; Warwick, 2003; Kaiser, 2005a). New recruits must be trained to treat objects in specific ways; what theorists will do with particular objects of theory is not dictated solely by the objects' own purported features. Indeed, the features or properties attributed to theoretical objects often owe more to pedagogical inculcation than anything else. This was especially the case for objects like the Brans-Dicke and Higgs fields, about which little to no observational evidence was available for decades, and for which no single theory compelled their union.

Unlike the positivists' neat and tidy picture of scientific theory progressing stepby-step in response to empirical data - and equally unlike the antipositivists' competing picture of top-down paradigms and antecedent theories determining all that can be described in scientific work - neither data nor theories forced young theorists such as Zee, Smolin, Steinhardt, Turner, and Kolb to begin studying the Brans-Dicke and Higgs fields as similar species of theoretical object. ${ }^{37}$ On the one hand, most particle theorists' attention turned to objects like the Brans-Dicke field just at the moment when astrophysical measurements seemed to weigh most strongly against Brans-Dicke gravitation. On the other hand, these same young theorists began to tinker with uniting the Higgs and Brans-Dicke fields independent of a coherent theory of high-energy earlyuniverse interactions that - only in retrospect - has been taken to be the primary motivation for these types of investigations. 
As these examples show, most of theorists' daily practices revolve around the skilled manipulation of theoretical tools and objects. These practices must be practiced: no student enters the research frontier based only on a casual perusal of articles or textbooks. Rather, apprentice-scientists must spend considerable effort - often over several years - learning the tricks of the trade, practicing how to construct do-able problems and work toward their solution with a specific set of methods. As Thomas Kuhn emphasized long ago, and as several scholars in science studies have recently elaborated upon, recognizing the salience of certain objects of theory and developing facility with a cache of techniques for their study are the end-products of pedagogy. Neither activity occurs on its own, and neither stands outside the active choices and decisions that scientists must always make about what topics their students should study, with what means, and toward what ends. Every aspect of new recruits' training depends on the outcome of these pedagogical decisions; and these decisions, in turn, rarely follow a strict logic of scientific development independent of broader institutional and educational considerations (Kuhn, 1962; Olesko, 1991; Geisen \& Holmes, 1993; Warwick, 2003; Kaiser, 2005a, 2005b).

Following the objects of theory around thus offers one way to combine insights from an older Mertonian tradition of the sociology of science with more recent work in a constructivist vein. Institutions and infrastructure - features that are obsessively quantified and measured in the tradition of 'scientometrics' - matter deeply to the modern sciences. Trends that often extend beyond an isolated laboratory or two can easily be missed if the focus remains exclusively on the hyper-local. Yet these institutional trends themselves are rarely the whole story: budget lines and enrollment patterns never interpret themselves; structural changes always underdetermine scientists' reactions to them. Hence the need to unite these numbers-rich analyses with careful attention to debates over what should count as a worthy topic of scientific research - and, even more important, debates over what should count as an appropriate topic or method 
for pedagogical propagation. In this way, we may use the objects of theory as tracers, mapping intertwined epistemic and social relations.

Tracking physicists' treatments of the Brans-Dicke field and the Higgs field illuminates broader changes in the recent history of physics, especially the processes by which particle cosmology became a flourishing field. While the much-vaunted changes in particle theory during the mid-1970s were clearly important in helping to establish particle cosmology, they are far from the whole story. Vast institutional changes shook the discipline, especially in the United States, during the late 1960s and early 1970s. These major changes in institutions and infrastructure led in turn to concrete changes in training. In the wake of the rapid collapse of the first 'Cold War bubble', pedagogical norms that had long been taken for granted were called into question. Particle theorists were no longer trained in the same hyper-specialized way that had marked the earlier Cold War decades. Only after these pedagogical changes began to take root did a new generation of theorists in the United States find it 'natural' to study the two objects $-\varphi_{B D}$ and $\varphi_{H}-$ as part of a common project. Much like theorists' tools, the objects of theory evolve in tandem with changes in their handlers' training. The objects and the young theorists who study them must be forged as part of the same pedagogical process. 


\section{Notes}

It is a pleasure to thank David Lucsko and Sameer Shah for research assistance; Carl Brans, the late Robert Dicke, Lee Smolin, and Anthony Zee for discussions; and Michael Gordin, Mike Lynch, Cyrus Mody, Sam Schweber, Christopher Smeenk, Spencer Weart, and four anonymous referees for helpful suggestions on an earlier draft. The following abbreviations are used: $N B L$, Niels Bohr Library, American Institute of Physics, College Park, Maryland; LIS, Leonard I. Schiff papers, call number SC220, Stanford University Archives, Palo Alto, California; PSC, Physics Survey Committee, United States National Academy of Sciences.

${ }^{1}$ On budgets, see http://www.cfo.doe.gov/budget, http://dellweb.bfa.nsf.gov, and http://www.nasa.gov/about/budget/index.html. Rate of preprints derived from data available at http://www.arXiv.org. (All accessed 7 November 2004.)

${ }^{2}$ For a broadly-accessible introduction to Mach's principle, see Will (1993 [1986]: 149-53); see also Barbour \& Pfister (1995). On Mach's influence on Einstein, see esp. Holton (1988 [1973]); Hoefer (1994); and Janssen (2005).

${ }^{3}$ A scalar field takes exactly one value at every point in space and time. For example, one could measure the temperature at every position in a room and repeat the measurements over time, and represent the measurements by a scalar field, $T$, of temperature. Electric and magnetic fields are examples of more complicated fields: these are vector fields, which carry several distinct components at every point in space and time (such as the value of the field in the $x$ direction, in the $y$ direction, and in the $z$ direction). In general relativity, the gravitational field is represented by a still more complicated type of field: a tensor field, which has an entire matrix of components at every point in space and time.

${ }^{4}$ The idea that $G$ could vary over time or space was tied to Paul Dirac's 'largenumber hypothesis', and put into concrete form by Pascual Jordan in 1955; to this day, 
the Brans-Dicke field is sometimes referred to as the Jordan-Brans-Dicke field. The main difference between Jordan's and Brans and Dicke's formulations was that Jordan included the energy of his scalar field in the energy-momentum tensor for ordinary matter, whereas Brans and Dicke kept these separate, treating the energy-momentum tensor of ordinary matter as the source for their scalar field's equation of motion. On Dirac's and Jordan's work, see Jordan (1955, 1959); and Kragh (1982). Brans learned of Jordan's work only after having completed most of his dissertation research. Carl H. Brans, email to the author, 30 July 1997.

${ }^{5}$ Several of the researchers, including Goldstone, came to the problem principally as solid-state theorists rather than particle theorists. Peter Higgs later explained that he would have found his solution to the particle theory problem more quickly if he had not been so ignorant of the solid-state theorists' work: Peter Higgs interview with Andrew Pickering, 21 October 1977, session I. Copies of the audio tapes are available in NBL.

${ }^{6}$ Higgs's main contribution was to show that all of the fields acquire some nonzero mass. Goldstone had proven that whenever a continuous symmetry is spontaneously broken, there must emerge a massless scalar field (later dubbed the 'Goldstone boson'). What Higgs demonstrated was that in gauge field theories, not only do the gauge fields acquire mass from their interactions with the Higgs field, but all of the remaining massless scalar fields can be gauged away, leaving only massive particles in the final particle spectrum.

${ }^{7}$ The papers represented in Figure 2 are all those that cited either the original 1961 Brans-Dicke paper (left), or all those that cited either of Higgs's 1964 papers or his 1966 paper; often in this period physicists cited some or all of the Higgs papers together. 'Renowned' is now a technical term among high-energy physicists. The high-energy physics literature database maintained by the Stanford Linear Accelerator Laboratory (SLAC) sorts articles according to their cumulative citations: unknown papers (0 
citations); less-known papers (1-9); known papers (10-49); well-known papers (50-99); famous papers (100-499); and renowned papers (500 or more citations). As of 11 November 2004, this database includes 615 papers that have received 500 or more citations, out of its database of more than 550,000 physics articles. See http://www.slac.stanford.edu/spires/hep.

${ }^{8}$ One might object that the two fields were not necessarily so similar: the BransDicke field was strictly classical, whereas the Higgs field was a quantum field. Yet in all of the early publications on the particle theory side, the essentials of spontaneous symmetry breaking were always discussed first in terms of a classical scalar field, $\varphi$; only later, once the main transformation properties and interactions of $\varphi$ were in place, did particle theorists quantize the system and begin treating $\varphi$ like an operator field. Thus at the level of theoretical manipulations, both the Brans-Dicke field and the Higgs field were treated as essentially classical - which remains true to this day in pedagogical treatments of the Higgs mechanism. Higgs himself made this clear throughout his lengthy 1966 article: (Higgs, 1966, esp. 1158-9). See also Kane (1987: chap. 8); Frampton (1987: chap. 1); and Weinberg (1995, vol 2: chaps. 19-20).

${ }^{9}$ When I asked Prof. Dicke if he had been aware at the time of the work by Goldstone, Higgs, et al. on introducing a new scalar field to explain the origin of mass, he replied, 'No, I'm afraid not. And I'm sure it was the same on the other side.' Robert H. Dicke, interview with the author, Princeton, 10 March 1995.

${ }^{10}$ On the Landau-Lifshitz textbook, see Kaiser (2000), 611-16; and Hall (2005); on general relativity in Cambridge, see Warwick (2003), chap. 9, and Prof. J. S. R. Chisholm, emails to the author, 19 July 2000, 27 Sep 2000, and 11 Mar 2003. See also the 'International Society on General Relativity and Gravitation', Records, 1961-1982, call number AR94 in NBL. 
${ }^{11}$ Data on citations to these articles are from Science Citation Index (1961-), s.v. 'Alpher, R. A.' and 'Gamow, G.'

${ }^{12}$ During the early 1960 s, not one physics department in the United States required coursework in general relativity for its graduate students, and most offered no courses on the subject at all. See Appendix 15 of W. C. Kelly, 'Survey of education in physics in universities of the United States', 1 Dec 1962, in American Institute of Physics Education and Manpower records, box 9, call number AR215 in NBL.

${ }^{13}$ Among physicists' accounts, see Pagels (1982), 275-77; Davies (1984), 159-60; Gribbin (1986), 293, 307, 312, 321, 345; Adair (1987), 357; Guth (1989), 105-6; Kolb (1996), 277-80; and Greene (1999), 177. Among science writers, see Bartusiak (1986), 227; Ferris (1988), 336-37; and Overbye (1991), 204, 234. Among philosophers, historians, and sociologists, see Pickering (1984), 387; Lightman \& Brawer (1990), 41; and Zinkernagel (2002), 496-97.

${ }^{14}$ Data on cosmology publications worldwide come from index entries (under 'cosmology') in Physics Abstracts; data on cosmology publications in the Physical Review come from keyword searches in the journal's on-line archive, available at http://prola.aps.org. The keywords included 'cosmology', 'cosmological', and 'universe'.

${ }^{15}$ For example, Georgi \& Glashow (1974) received fewer than fifty citations worldwide per year between 1974 and 1978, rapidly rising to more than two hundred citations per year starting in 1980. Science Citation Index (1961-), s.v. 'Georgi, H.' Anthony Zee similarly recalls that GUTs received little attention from particle theorists until the very end of the 1970s: Zee (1989), 117.

${ }^{16}$ Steigman (1979) makes passing reference in his introduction to the new work on grand unification (313-14), but explicitly labels GUT-related cosmological ideas 'beyond the scope of this review' (328; see also 336). 
${ }^{17}$ Rasmussen (1997) also uses the stock-market metaphor of a 'bubble' to describe postwar patterns in American science.

${ }^{18}$ Figure 5 is based on data in the appendices of Adkins (1975: 278-81), with later data (for the period 1970-81) from PSC (1986: 98). The number of physics Ph.D.s granted per year in the United States crested in 1971, with 1681 Ph.D.s granted in that year alone.

${ }^{19}$ On falling funds, see PSC (1986: 120); on the job crunch, see 'placement service register' materials, ca. 1970-71, in the American Institute of Physics Education and Manpower Division, box 13, Folders 'Placement literature' and 'Placement service advisory committee', call number AR215 in NBL; see also Kaiser (2002: 151).

${ }^{20}$ PSC (1972, vol. 1: 364); PSC (1986: 98). On some of the new developments in gravitation and cosmology during this period, see Will (1993 [1986]); Kragh (1996); and Collins (2004).

${ }^{21}$ Data on dissertations on gravitation and cosmology come from Dissertation Abstracts, available via http://www.oclc.org.

${ }^{22}$ On the interplay of market forces and the scientific textbook trade, see also García-Belmar et al. (2005); and Hall (2005).

${ }^{23}$ Kaiser (1998), 321-22; W. C. Kelly, 'Survey of education'.

${ }^{24}$ Quotation from Arthur Beiser (physicist at New York University) to Malcolm Johnson (editor at McGraw-Hill), 14 April 1959, in LIS, Box 12, Folder 'Yilmaz: Relativity'. See also the similar correspondence throughout Schiff's files during this period. Schiff was the series editor for the highly successful 'International Series in Pure and Applied Physics' (a textbook series published by McGraw-Hill), and had to work hard to convince the publishers to take risks on a few new textbooks on gravitation (a subject to which Schiff had already turned some of his attention): $L I S$, Box 12, 'Schiffer: Gen. Rel.'; Box 12, 'Tauber: Gen. Rel.'; and Box 13, 'Yilmaz: Relativity'. 
${ }^{25}$ These figures come from keyword and call-number searches in the on-line catalog of the Library of Congress: http://www.loc.gov.

${ }^{26}$ Richard Feynman, 'Lectures on gravitation', mimeographed lecture notes prepared by Fernando B. Morinigo and William G. Wagner, based on Feynman's graduate course at Caltech in 1962-63, and distributed widely beginning in 1971; these were later published as Feynman (1995). Mimeographed copies of Feynman's notes found their way into at least fifty university libraries across the United States, ranging from small liberal arts colleges like Wellesley College in Massachusetts and Reed College in Oregon to major training centers like Harvard, Stanford, Berkeley, Yale, Princeton, the University of Maryland, and many others. (Based on information in the WorldCat on-line catalog: http://www.oclc.org.) On Feynman's idiosyncratic approach to gravitation in these notes, see Kaiser (1998: 329-31). See also Papapetrou (1974).

${ }^{27}$ Many particle theorists recall that Weinberg's textbook was particularly helpful when trying to learn about general relativity and cosmology. Weinberg himself came from a particle theory background, and his notation and style of argument seemed much less foreign for fellow particle theorists than those used in many other relativity textbooks. See the interviews in Lightman \& Brawer (1990); Anthony Zee likewise emphasized the point in his interview with me: Anthony Zee, telephone interview, 16 May 2005.

${ }^{28}$ Note that both Zee and Smolin parameterized their gravitational equations slightly differently than Brans and Dicke did. They followed the usual particle-theory convention of giving scalar fields the dimension of mass. In these units, Newton's gravitational constant is proportional to $1 /(\text { mass })^{2}$, and hence they set $G$ equal to the inverse square of their scalar field, rather than to the inverse of the field as Brans and Dicke had done. 
${ }^{29}$ Zee (1989: 112); Anthony Zee, letter to John Wheeler, Feb 1977, included in the 'Wheeler Family Gathering', vol. 2 (a collection of reminiscences by Wheeler's former students), a copy of which may be found in NBL, call number AR167; and Anthony Zee, telephone interview with the author, 16 May 2005. Several other theorists of Zee's generation followed similarly meandering trajectories into particle cosmology. David Schramm, for example, had studied nuclear and particle physics as an undergraduate at MIT in the mid-1960s before completing his Ph.D. in gravitational theory with Kip Thorne at Caltech in 1971. See Schramm's interview in Lightman and Brawer (1990: 435-50). Alan Guth, meanwhile, completed his dissertation in particle theory at MIT in 1973 and struggled through a series of postdocs during the mid- and late 1970s, his personal fortunes mirroring those of mainstream particle theory in the United States. Guth's interest in cosmology was sparked by a chance encounter with Robert Dicke in 1978. Within months of that encounter, he turned his research focus squarely to particle cosmology; soon he achieved renown for his invention of inflationary cosmology, which has remained a central topic within particle cosmology to this day (see Guth, 1997, Chapter 1).

${ }^{30}$ For Klein's influence on Deser, see Deser (1957); on Deser's early interactions with Higgs, see Brown et al. (1997: 509). Boulware \& Deser (1972) was the first paper to cite both the Brans-Dicke work and the Higgs work. Klein's work from the 1920s on unifying gravitation with electromagnetism by positing the existence of an extra spatial dimension in the universe has returned to prominence; see Applequist et al. (1987); and Randall (2002). My thanks to Sam Schweber for bringing to my attention Deser's connections to Klein.

${ }^{31}$ Lee Smolin, interview with the author, 1 December 2004, at MIT. See also Smolin (1997: 7-8, 50); and Smolin (2004). For more on Coleman's Harvard course on general relativity, see Kaiser (1998), 331-33. 
${ }^{32}$ Quotations from Nadis (2004: 46). On the Stanford gravity discussion group, see the correspondence in LIS, Box 16, Folder, 'Discussion group: General relativity and astrophysics, 1970-71'.

${ }^{33}$ See 'DeWitt, Bryce S.', in Cattell (1982, vol. 2:618). Information on Austin's output of Ph.D.s comes from Dissertation Abstracts. On Kolb's early research, see Dicus \& Kolb (1977a, 1977b); Dicus, Kolb \& Teplitz (1977); Dicus et al. (1978a, 1978b).

${ }^{34}$ On further studies of the cosmological consequences of uniting $\varphi_{B D}$ with $\varphi_{H}$, see, for example, Kaiser (1994a, 1994b, 1995).

${ }^{35}$ On the debates over the Superconducting Supercollider, see esp. Kevles (1995); Riordan (2001). See also Lederman with Teresi (1993); and Wouk (2004).

${ }^{36}$ In the second edition of his otherwise excellent book, Clifford Will notes in one parenthetical sentence that '(Only a late-1980s flurry of interest in variants of the BransDicke theory in models of an 'inflationary' phase of the universe kept its flame flickering.)’ Will (1993 [1986]), 158. Such a characterization entirely misses what had already become routine by the late 1980 s and continues to be the dominant set of assumptions throughout most of particle cosmology about alterations to canonical Einsteinian gravity during the early phases of the universe.

${ }^{37}$ See Galison (1988), and Galison (1997: chap. 9). 


\section{References}

Accetta, F. S., D. J. Zoller \& M. S. Turner (1985) 'Induced-Gravity Inflation', Physical Review D 31: 3046-3051.

Adair, Robert (1987) The Great Design: Particles, Fields, and Creation (New York: Oxford University Press).

Adkins, Douglas (1975) The Great American Degree Machine: An Economic Analysis of the Human Resource Output of Higher Education (Berkeley, CA: Carnegie Institution).

Adler, Ronald, Maurice Bazin \& Menahim Schiffer (1975 [1965]) Introduction to General Relativity, 2nd edn (New York: McGraw-Hill).

Alpher, Ralph A. (1948) 'A Neutron Capture Theory of the Formation and Relative Abundance of the Elements', Physical Review 74: 1577-1589.

Alpher, Ralph A., Hans Bethe \& George Gamow (1948) 'The Origin of Chemical Elements', Physical Review 73: 803-804.

Alpher, Ralph A. \& Robert Herman (1948) 'On the Relative Abundance of the Elements', Physical Review 74: 1737-1742.

Alpher, Ralph A. \& Robert Herman (1949) 'Remarks on the Evolution of the Expanding Universe', Physical Review 75: 1089-1095.

Alpher, Ralph A. \& Robert Herman (1950) 'Theory of the Origin and Relative Abundance Distribution of the Elements', Reviews of Modern Physics 22: 153212.

Alpher, Ralph A., Robert Herman \& George Gamow (1948) 'Thermonuclear Reactions in the Expanding Universe', Physical Review 74: 1198-1199.

Applequist, Thomas, Alan Chodos \& Peter G. O. Freund (eds) (1987) Modern KaluzaKlein Theories (Menlo Park, CA: Addison-Wesley).

Arabatzis, Theodore (1995) The Electron: A Biographical Sketch of a Theoretical Entity. Ph.D. Thesis, Princeton University.

Arabatzis, Theodore (1996) 'Rethinking the "Discovery" of the Electron', Studies in History and Philosophy of Science 27: 405-435.

Barbour, Julian \& Herbert Pfister (eds) (1995) Mach's Principle: From Newton's Bucket to Quantum Gravity (Boston: Birkhäuser).

Bartusiak, Marcia (1986) Thursday's Universe: A Report from the Frontier on the Origin, Nature, and Destiny of the Universe (New York: Times Books).

Bergmann, Peter (1976 [1942]) Introduction to the Theory of Relativity, 2nd edn (New York: Dover).

Boulware, David \& Stanley Deser (1972) 'Can Gravitation Have a Finite Range?' Physical Review D 6: 3368-3382.

Brans, Carl H. (1961) Mach's Principle and a Varying Gravitational Constant. Ph.D. Thesis, Princeton University.

Brans, Carl H. \& Robert H. Dicke (1961) 'Mach's Principle and a Relativistic Theory of Gravitation', Physical Review 124: 925-935.

Brown, Laurie \& Helmut Rechenberg (1996) The Origin of the Concept of Nuclear Forces (Philadelphia, PA: Institute of Physics Publishing). 
Brown, Laurie, Robert Brout, Tian Yu Cao, Peter Higgs \& Yoichiro Nambu (1997) 'Panel Session: Spontaneous Breaking of Symmetry', in L. Hoddeson, L. Brown, M. Riordan \& M. Dresden (eds), The Rise of the Standard Model (New York: Cambridge University Press): 478-522.

Brown, Laurie \& Tian Yu Cao (1991) 'Spontaneous Breakdown of Symmetry: Its Rediscovery and Integration into Quantum Field Theory', Historical Studies in the Physical and Biological Sciences 21: 211-235.

Brown, Laurie, Max Dresden \& Lillian Hoddeson (eds) (1989) Pions to Quarks: Particle Physics in the 1950s (New York: Cambridge University Press).

Cattell, Jacques (ed) (1982) American Men and Women of Science: Physical and Biological Sciences, 15th edn (New York: R. R. Bowker).

Chandrasekhar, Subramanyan (1939) An Introduction to the Study of Stellar Structure (Chicago, IL: University of Chicago Press).

Chandrasekhar, Subramanyan (1942) Principles of Stellar Dynamics (Chicago, IL: University of Chicago Press).

Choi, Charles (2004) 'The Phantom Menace', Scientific American 291 (October 2004): 41-44.

Chudnovskii, E. M. (1978) 'Spontaneous Breaking of Conformal Invariance and the Higgs Mechanism', Theoretical and Mathematical Physics 35: 538-539.

Collins, Harry (2004) Gravity's Shadow: The Search for Gravitational Waves (Chicago, IL: University of Chicago Press).

Daston, Lorraine (ed.) (2000) Biographies of Scientific Objects (Chicago, IL: University of Chicago Press).

Davies, Paul (1984) God and the New Physics (New York: Penguin).

Deser, Stanley (1957) 'General Relativity and the Divergence Problem in Quantum Field Theory', Reviews of Modern Physics 29: 417-423.

Dicke, R. H. \& H. Mark Goldenberg (1967) 'Solar Oblateness and General Relativity', Physical Review Letters 18: 313-316.

Dicke, Robert H. (1964a) 'The Many Faces of Mach', in H.-Y. Chiu and W. Hoffmann (eds), Gravitation and Relativity (New York: W. A. Benjamin): 121-141.

Dicke, Robert H. (1964b) The Theoretical Significance of Experimental Relativity (New York: Gordon and Breach).

Dicus, Duane \& Edward W. Kolb (1977a) 'Stellar Energy-Loss Rates Due to $S, P$, or $T$ Neutral Currents', Physical Review D 15: 977-982.

Dicus, Duane \& Edward W. Kolb (1977b) 'Reaction Rate, Weak Corrections, and Background to $e^{+} e^{-} \rightarrow \pi^{0} \gamma, \eta \gamma^{\prime}$, Physical Review D 15: 1286-1292.

Dicus, Duane, Edward W. Kolb \& Vigdor Teplitz (1977) 'Cosmological Upper Bound on Heavy-Neutrino Lifetimes', Physical Review Letters 39: 168-171.

Dicus, Duane, Edward W. Kolb, Vigdor Teplitz \& Robert Wagoner (1978a) 'Limits from Primordial Nucleosynthesis on the Properties of Massive Neutral Leptons', Physical Review D 17: 1529-1538.

Dicus, Duane, Edward W. Kolb, Vigdor Teplitz \& Robert Wagoner (1978b) 'Astrophysical Bounds on the Masses of Axions and Higgs Particles', Physical Review D 18: 1829-1834.

Dvali, Georgi (2004) 'Out of the Darkness', Scientific American 290 (February): 68-75. 
Eisenstadt, Jean (1989) 'The Low Water Mark of General Relativity, 1925-1955', in D. Howard \& J. Stachel (eds), Einstein and the History of General Relativity (Boston: Birkhäuser): 277-292.

Englert, F., E. Gunzig, C. Truffin \& P. Windey (1975) 'Conformal Invariant General Relativity with Dynamical Symmetry Breakdown', Physics Letters B 57: 73-77.

Ferris, Timothy (1988) Coming of Age in the Milky Way (New York: Anchor).

Feynman, Richard (1995) Lectures on Gravitation (Reading, MA: Addison-Wesley). Fock, Vladimir (1959 [1955]) The Theory of Space-Time and Gravitation, trans. N. Kemmer (New York: Pergamon).

Frampton, Paul H. (1987) Gauge Field Theories (Reading, MA: Benjamin Cummings).

Fujii, Yasunori (1974) 'Scalar-Tensor Theory of Gravitation and Spontaneous Breakdown of Scale Invariance’, Physical Review D 9: 874-876.

Galison, Peter (1987) How Experiments End (Chicago, IL: University of Chicago Press). Galison, Peter (1988) 'History, Philosophy, and the Central Metaphor', Science in Context 2: 197-212.

Galison, Peter (1997) Image and Logic: A Material Culture of Microphysics (Chicago, IL: University of Chicago Press).

Galison, Peter \& Andrew Warwick (eds) (1998) The Cultures of Theory. Published in Studies in History and Philosophy of Modern Physics 29: 287-434.

Gamow, George (1948a) 'The Origin of Elements and the Separation of Galaxies', Physical Review 74: 505-506.

Gamow, George (1948b) 'The Evolution of the Universe', Nature 162: 680-682.

Gamow, George (1949) 'On Relativistic Cosmogony', Reviews of Modern Physics 21: 367-373.

García-Belmar, Antonio, José Ramón Bertomeu-Sánchez \& Bernadette BensaudeVincent (2005) 'The Power of Didactic Writings: French Chemistry Textbooks of the Ninetheenth Century', in D. Kaiser (ed.), Pedagogy and the Practice of Science (Cambridge, MA: MIT Press): 219-252.

Geisen, Gerald L. \& Frederic L. Holmes (eds) (1993) Research Schools: Historical Reappraisals, Osiris 8: 3-248.

Georgi, Howard \& Sheldon Glashow (1974) 'Unity of All Elementary Particle Forces', Physical Review Letters 32: 438-441.

Goldstone, Jeffrey (1961) 'Field Theories with "Superconductor” Solutions', Nuovo Cimento 19: 154-164.

Greene, Brian (1999) The Elegant Universe: Superstrings, Hidden Dimensions, and the Quest for the Ultimate Theory (New York: Norton).

Gribbin, John (1986) In Search of the Big Bang: Quantum Physics and Cosmology (New York: Bantam).

Gross, David J. \& Frank Wilczek (1973a) 'Ultraviolet Behavior of Nonabelian Gauge Theories', Physical Review Letters 30: 1343-1346.

Gross, David J. \& Frank Wilczek (1973b) 'Asymptotically Free Gauge Theories, I,' Physical Review D 8: 3633-3652.

Gross, David J. \& Frank Wilczek (1974) 'Asymptotically Free Gauge Theories, II', Physical Review D 9: 980-993. 
Guth, Alan (1989) 'Starting the Universe: The Big Bang and Cosmic Inflation', in J. Cornell (ed.), Bubbles, Voids, and Bumps in Time: The New Cosmology (New York: Cambridge University Press): 105-146.

Guth, Alan (1997) The Inflationary Universe: The Quest for a New Theory of Cosmic Origins (Reading, MA: Addison-Wesley).

Guth, Alan \& David Kaiser (2005) 'Inflationary Cosmology: Exploring the Universe from the Smallest to the Largest Scales', Science 307 (11 February): 884-890.

Hacking, Ian (1983) Representing and Intervening (New York: Cambridge University Press).

Hall, Karl (2005) "Think Less about Foundations": A Short Course on Landau and Lifshitz's Course of Theoretical Physics', in D. Kaiser (ed.), Pedagogy and the Practice of Science: Historical and Contemporary Perspectives (Cambridge, MA: MIT Press): 253-286.

Hawking, Stephen \& George Ellis (1973) The Large-Scale Structure of Space-Time (New York: Cambridge University Press).

Higgs, Peter W. (1964a) 'Broken Symmetries, Massless Particles, and Gauge Fields', Physics Letters B 12: 132-133.

Higgs, Peter W. (1964b) 'Broken Symmetries and the Masses of Gauge Bosons', Physical Review Letters 13: 508-509.

Higgs, Peter W. (1966) 'Spontaneous Symmetry Breakdown without Massless Bosons', Physical Review 145: 1156-1163.

Hill, H. A., P. D. Clayton, D. L. Patz \& A. W. Healy (1974) 'Solar Oblateness, Excess Brightness, and Relativity', Physical Review Letters 33: 1497-1500.

Hoddeson, Lillian, Laurie Brown, Michael Riordan \& Max Dresden (eds) (1997) The Rise of the Standard Model: Particle Physics in the 1960s and 1970s (New York: Cambridge University Press).

Hoefer, Carl (1994) 'Einstein's Struggle for a Machian Gravitation Theory', Studies in History and Philosophy of Science 25: 287-335.

Holman, R., E. W. Kolb \& Y. Wang (1990) 'Gravitational Couplings of the Inflaton in Extended Inflation', Physical Review Letters 65: 17-20.

Holton, Gerald (1988 [1973]) 'Mach, Einstein, and the Search for Reality', in G. Holton, Thematic Origins of Scientific Thought: Kepler to Einstein, 2nd edn (Cambridge, MA: Harvard University Press): chap. 7.

Jammer, Max (1961) Concepts of Mass in Classical and Modern Physics (Cambridge, MA: Harvard University Press).

Jammer, Max (2000) Concepts of Mass in Contemporary Physics and Philosophy (Princeton, NJ: Princeton University Press).

Janssen, Michel (2005) 'Of Pots and Holes: Einstein's Bumpy Road to General Relativity', Annalen der Physik 14 (Supplement): 58-85.

Jordan, Pascual (1955) Schwerkraft und Weltall (Braunschweig: Vieweg).

Jordan, Pascual (1959) 'The Present State of Dirac's Cosmological Hypothesis', Zeitschrift für Physik 157: 112-121.

Kaiser, David (1994a) 'Constraints in the Context of Induced-Gravity Inflation', Physical Review D 49: 6347-6353.

Kaiser, David (1994b) 'Induced-Gravity Inflation and the Density Perturbation Spectrum', Physics Letters B 340: 23-28. 
Kaiser, David (1995) 'Primordial Spectral Indices from Generalized Einstein Theories', Physical Review D 53: 4295-4306.

Kaiser, David (1998) 'A $\psi$ is just a $\psi$ ? Pedagogy, Practice, and the Reconstitution of General Relativity, 1942-1975', Studies in History and Philosophy of Modern Physics 29: 321-338.

Kaiser, David (2000) Making Theory: Producing Physics and Physicists in Postwar America. Ph.D. Thesis, Harvard University.

Kaiser, David (2002) 'Cold War Requisitions, Scientific Manpower, and the Production of American Physicists after World War II', Historical Studies in the Physical and Biological Sciences 33: 131-159.

Kaiser, David (2005a) Drawing Theories Apart: The Dispersion of Feynman Diagrams in Postwar Physics (Chicago, IL: University of Chicago Press).

Kaiser, David (ed.) (2005b) Pedagogy and the Practice of Science: Historical and Contemporary Perspectives (Cambridge, MA: MIT Press).

Kaiser, David, Kenji Ito \& Karl Hall (2004) 'Spreading the Tools of Theory: Feynman Diagrams in the United States, Japan, and the Soviet Union', Social Studies of Science 34: 879-922.

Kane, Gordon (1987) Modern Elementary Particle Physics (Redwood City, CA: Addison-Wesley).

Kaufmann, William (1976 [1973]) Relativity and Cosmology, 2nd edn (New York: Harper and Row).

Kennefick, Daniel (2000) 'Star Crushing: Theoretical Practice and the Theoreticians' Regress', Social Studies of Science 30: 5-40.

Kevles, Daniel (1995 [1978]) The Physicists: The History of a Scientific Community in Modern America, 3rd edn (Cambridge, MA: Harvard University Press).

Kevles, Daniel (1995) 'Preface, 1995: The Death of the Superconducting Super Collider in the Life of American Physics', in D. Kevles, The Physicists: The History of a Scientific Community in Modern America, 3rd edn (Cambridge, MA: Harvard University Press): ix-xlii.

Klein, Ursula (ed.) (2001) Tools and Modes of Representation in the Laboratory Sciences (Boston, MA: Kluwer).

Klein, Ursula (2003) Experiments, Models, Paper Tools: Cultures of Organic Chemistry in the Nineteenth Century (Stanford: Stanford University Press).

Kolb, Edward W. \& Michael S. Turner (1990) The Early Universe (Reading, MA: Addison-Wesley).

Kolb, Rocky [Edward W.] (1996) Blind Watchers of the Sky: The People and Ideas that Shaped our View of the Universe (Reading, MA: Addison-Wesley).

Kragh, Helge (1982) 'Cosmo-Physics in the Thirties: Towards a History of Dirac Cosmology', Historical Studies in the Physical Sciences 13: 70-108.

Kragh, Helge (1996) Cosmology and Controversy: The Historical Development of Two Theories of the Universe (Princeton, NJ: Princeton University Press).

Kragh, Helge (1999) Quantum Generations: A History of Physics in the Twentieth Century (Princeton, NJ: Princeton University Press).

Krauss, Lawrence M. \& Michael S. Turner (2004) ‘A Cosmic Conundrum', Scientific American 291 (September): 70-77. 
Kuhn, Thomas S. (1962) The Structure of Scientific Revolutions (Chicago: University of Chicago Press).

La, D. \& P. J. Steinhardt (1989) 'Extended Inflationary Cosmology', Physical Review Letters 62: 376-378.

Landau, Lev \& Evgenii Lifshitz (1951 [1941]) The Classical Theory of Fields, trans. Morton Hammermesh (Cambridge, MA: Addison-Wesley).

Landau, Lev \& Evgenii Lifshitz (1971) The Classical Theory of Fields, 3rd edn (New York: Pergamon).

Landau, Lev \& Evgenii Lifshitz (1975) The Classical Theory of Fields, 4th edn (New York: Pergamon).

Lederman, Leon with Dick Teresi (1993) The God Particle: If the Universe is the Answer, What is the Question? (New York: Houghton Mifflin).

Lightman, Alan \& Roberta Brawer (eds) (1990) Origins: The Lives and Worlds of Modern Cosmologists (Cambridge, MA: Harvard University Press).

Lightman, Alan, William Press, Richard Price \& Saul Teukolsky (1975) Problem Book in Relativity and Gravitation (Princeton, NJ: Princeton University Press).

Lloyd, Seth \& Y. Jack Ng (2004) 'Black Hole Computers', Scientific American 291 (November): 52-61.

MacKenzie, Donald (2003) 'An Equation and its Worlds: Bricolage, Exemplars, Disunity, and Performativity in Financial Economics', Social Studies of Science 33: 831-868.

Matsuki, Takayuki (1978) 'Effects of the Higgs Scalar on Gravity', Progress of Theoretical Physics 59: 235-241.

Minkowski, Peter (1977) 'On the Spontaneous Origin of Newton's Constant', Physics Letters B 71: 419-421.

Misner, Charles, Kip Thorne \& John Wheeler (1973) Gravitation (San Francisco, CA: W. H. Freeman).

Nadis, Steve (2004) ‘The Lost Years of Michael Turner', Astronomy 32 (April 2004): 44-49.

Olesko, Kathryn (1991) Physics as a Calling: Discipline and Practice in the Königsberg Seminar for Physics (Ithaca, NY: Cornell University Press).

Oppenheimer, J. R. \& Robert Serber (1938) 'On the Stability of Stellar Neutron Cores', Physical Review 54: 540.

Oppenheimer, J. R. \& H. Snyder (1939) 'On Continued Gravitational Contraction', Physical Review 56: 455-459.

Oppenheimer, J. R. \& G. M. Volkoff (1939) 'On Massive Neutron Cores', Physical Review 55: 374-381.

Overbye, Dennis (1991) Lonely Hearts of the Cosmos: The Story of the Scientific Quest for the Secret of the Universe (New York: Harper Collins).

Pagels, Heinz (1982) The Cosmic Code: Quantum Physics as the Language of Nature (New York: Bantam).

Papapetrou, Achilleus (1974) Lectures on General Relativity (Boston: Reidel).

Pati, Jogesh \& Abdus Salam (1973) 'Unified Lepton-Hadron Symmetry and a Gauge Theory of the Basic Interactions', Physical Review D 8: 1240-1251.

Pauli, Wolfgang (1958 [1921]) Theory of Relativity, trans. G. Field (New York: Pergamon). 
Peebles, P. J. E. (1971) Physical Cosmology (Princeton, NJ: Princeton University Press).

Pickering, Andrew (1984) Constructing Quarks: A Sociological History of Particle Physics (Chicago, IL: University of Chicago Press).

Pickering, Andrew \& Adam Stephanides (1992) 'Constructing Quaternions: On the Analysis of Conceptual Practice', in A. Pickering (ed.), Science as Practice and Culture (Chicago, IL: University of Chicago Press): 139-167.

Politzer, H. David (1973) 'Reliable Perturbative Results for Strong Interactions?' Physical Review Letters 30: 1346-1349.

Politzer, H. David (1974) ‘Asymptotic Freedom: An Approach to Strong Interactions', Physics Reports 14: 129-180.

Polkinghorne, John (1989) Rochester Roundabout: The Story of High Energy Physics (San Francisco, CA: W. H. Freeman).

PSC (1966) Physics: Survey and Outlook (Washington, DC: National Academy of Sciences).

PSC (1972) Physics in Perspective (Washington, DC: National Academy of Sciences).

PSC (1986) Physics Through the 1990s: An Overview (Washington, DC: National Academy Press).

Randall, Lisa (2002) 'Extra Dimensions and Warped Geometries', Science 296 (24 May 2002): 1422-1427.

Rasmussen, Nicholas (1997) 'The Midcentury Biophysics Bubble: Hiroshima and the Biological Revolution in America, Revisited', History of Science 35: 245-293.

Reasenberg, R. D. et al. (1979) 'Viking Relativity Experiment: Verification of Signal Retardation by Solar Gravity’, Astrophysical Journal 234: L219-221.

Richman, Sam (1996) 'Resolving Discordant Results: Modern Solar Oblateness Experiments', Studies in History and Philosophy of Modern Physics 27: 1-22.

Riordan, Michael (2001) 'A Tale of Two Cultures: Building the Superconducting Supercollider, 1988-93', Historical Studies in the Physical and Biological Sciences 32: 125-144.

Schramm, David N. (1978) 'Cosmology and New Particles', in P. A. Schreiner, G. H. Thomas, and A. B. Wicklund (eds), Particles and Fields, 1977 (New York: American Institute of Physics): 87-101.

Schweber, Silvan S. (1989) 'Some Reflections on the History of Particle Physics in the 1950s', in L. Brown, M. Dresden \& L. Hoddeson (eds), Pions to Quarks:

Particle Physics in the 1950s (New York: Cambridge University Press): 668-693.

Science Citation Index (1961-) (Philadelphia, PA: Institute for Scientific Information).

Smolin, Lee (1979) 'Towards a Theory of Spacetime Structure at Very Short Distances', Nuclear Physics B 160: 253-268.

Smolin, Lee (1997) The Life of the Cosmos (New York: Oxford University Press).

Smolin, Lee (2004) 'A Strange Beautiful Girl in a Car', in J. Brockman (ed.), Curious Minds: How a Child Becomes a Scientist (New York: Random House): 71-78.

Srinivasan, G. (ed.) (1999) From White Dwarfs to Black Holes: The Legacy of S. Chandrasekhar (Chicago, IL: University of Chicago Press).

Steigman, Gary (1979) 'Cosmology Confronts Particle Physics', Annual Review of Nuclear and Particle Science 29: 313-337.

Tayler, R. J. (1980) 'Cosmology, Astrophysics, and Elementary Particle Physics', Reports on Progress in Physics 43: 253-299. 
Tolman, Richard C. (1934) Relativity, Thermodynamics, and Cosmology (Oxford: Clarendon Press).

Veneziano, Gabrielle (2004) 'The Myth of the Beginning of Time', Scientific American 290 (May): 54-63.

Warwick, Andrew (2003) Masters of Theory: Cambridge and the Rise of Mathematical Physics (Chicago, IL: University of Chicago Press).

Weinberg, Steven (1972) Gravitation and Cosmology: Principles and Applications of the General Theory of Relativity (New York: Wiley).

Weinberg, Steven (1995) The Quantum Theory of Fields (New York: Cambridge University Press).

Weyl, Hermann (1950 [1919]) Space, Time, Matter, trans. Henry L. Brose (New York: Dover).

WGBH (1999) Time Travel (Cambridge, MA: WGBH/NOVA television).

WGBH (2000) Runaway Universe (Cambridge, MA: WGBH/NOVA television).

WGBH (2003) The Elegant Universe (Cambridge, MA: WGBH/NOVA television).

Will, Clifford M. (1993 [1981]) Theory and Experiment in Gravitational Physics, 2nd edn (New York: Cambridge University Press).

Will, Clifford M. (1993 [1986]) Was Einstein Right? Putting General Relativity to the Test, 2nd edn (New York: Basic Books).

Wouk, Herman (2004) A Hole in Texas (Boston: Little, Brown).

Zee, Anthony (1979) ‘Broken-Symmetric Theory of Gravity', Physical Review Letters 42: 417-421.

Zee, Anthony (1989) An Old Man's Toy: Gravity at Work and Play in Einstein's Universe (New York: Macmillan).

Zinkernagel, H. (2002) 'Cosmology, Particles, and the Unity of Science', Studies in History and Philosophy of Modern Physics 33: 493-516. 